\title{
ARTICLE
}

\section{Vulnerabilities in mIDH2 AML confer sensitivity to APL-like targeted combination therapy}

\author{
Vera Mugoni ${ }^{1}$, Riccardo Panella ${ }^{1}$, Giulia Cheloni ${ }^{1}$, Ming Chen ${ }^{1}$, Olga Pozdnyakova ${ }^{2}$, Dina Stroopinsky ${ }^{3}$, Jlenia Guarnerio ${ }^{1}$, \\ Emanuele Monteleone (iD ${ }^{1,4}$, Jonathan David Lee ${ }^{1}$, Lourdes Mendez ${ }^{1}$, Archita Venugopal Menon ${ }^{1}$, Jon Christopher Aster ${ }^{5}$, \\ Andrew A. Lane ${ }^{2}$, Richard Maury Stone ${ }^{5}$, Ilene Galinsky ${ }^{5}$, José Cervera Zamora ${ }^{6}$, Francesco Lo-Coco ${ }^{7,8}$, Manoj Kumar Bhasin ${ }^{9}$, \\ David Avigan ${ }^{3}$, Letizia Longo ${ }^{1}$, John Gerard Clohessy (D) ${ }^{1,10}$ and Pier Paolo Pandolfi (D)
}

\begin{abstract}
Although targeted therapies have proven effective and even curative in human leukaemia, resistance often ensues. IDH enzymes are mutated in $\sim 20 \%$ of human $\mathrm{AML}$, with targeted therapies under clinical evaluation. We here characterize leukaemia evolution from mutant IDH2 (mIDH2)-dependence to independence identifying key targetable vulnerabilities of $\mathrm{mIDH} 2$ leukaemia that are retained during evolution and progression from early to late stages. Mechanistically, we find that mIDH2 leukaemia are metastable and vulnerable at two distinct levels. On the one hand, they are characterized by oxidative and genotoxic stress, in spite of increased 1-carbon metabolism and glutathione levels. On the other hand, mIDH2 leukaemia display inhibition of LSD1 and a resulting transcriptional signature of all-trans retinoic acid (ATRA) sensitization, in spite of a state of suppressed ATRA signalling due to increased levels of PIN1. We further identify GSH/ROS and PIN1/LSD1 as critical nodes for leukaemia maintenance and the combination of ATRA and arsenic trioxide (ATO) as a key therapeutic modality to target these vulnerabilities. Strikingly, we demonstrate that the combination of ATRA and ATO proves to be a powerfully synergistic and effective therapy in a number of mouse and human mIDH1/2 leukemic models. Thus, our findings pave the way towards the treatment of a sizable fraction of human AMLs through targeted APL-like combinatorial therapies.
\end{abstract}

Cell Research (2019) 29:446-459; https://doi.org/10.1038/s41422-019-0162-7

\section{INTRODUCTION}

Mutations to isocitrate dehydrogenase (IDH1 and IDH2), key metabolic enzymes, are an important early event in a variety of tumor types. ${ }^{1-3}$ The mutant enzymes give rise to the oncometabolite 2-hydroxyglutarate (2-HG) that contributes to the oncogenic phenotype. ${ }^{4-6}$ Thus, extensive efforts have focused on the development of specific inhibitors targeting individual IDH mutants. ${ }^{7}$ The recent success of the $\mathrm{mIDH} 2$ targeting Enasidenib (AG-221) in clinical trials have led to its approval by the FDA for the treatment of relapsed or refractory acute myeloid leukaemia (AML) with an IDH2 mutation. ${ }^{8}$

However, resistance to single agent therapies is a frequent event ${ }^{9,10}$ and this is also the case for Enasidenib in the treatment of relapsed or refractory $\mathrm{mIDH} 2 \mathrm{AML}$, leading to disease progression. $^{10,11}$ Resistance mechanisms frequently highlight common vulnerabilities, and the identification of such vulnerabilities can prevent the emergence of resistance and facilitate treatment. ${ }^{11}$ How mIDH cancers adjust and evolve in response to treatment, or what vulnerabilities may be associated with mIDH remain largely unknown, although recent studies from $\mathrm{mIDH} 2$ patients treated with Enasidenib suggest that resistance may arise through a variety of mechanisms. ${ }^{11,12}$

In order to develop therapies that would facilitate the use of inhibitors to treat cancers harboring mutant IDH, we focused on identifying vulnerabilities that would be associated with the presence of a mIDH enzyme in acute myeloid leukaemia (AML).

Building on our previously published genetically engineered mouse models (GEMMs) for AML harboring a mIDH2 allele, ${ }^{13}$ and expanding on this using a variety of additional murine and cell line models, we evaluated what vulnerabilities underlie mIDH AML. In doing so we identified critical vulnerabilities that can be readily targeted for therapeutic benefit. On this basis, we show that APLlike ATRA/ATO combination is powerfully and exquisitely effective

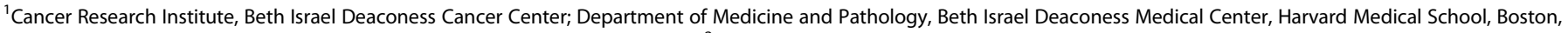

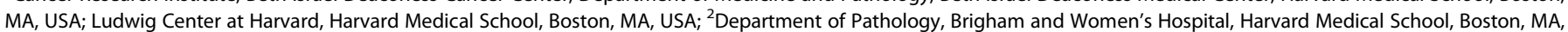

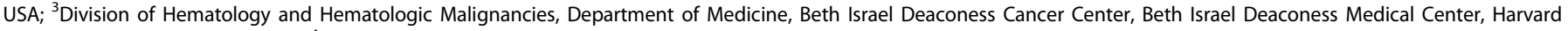

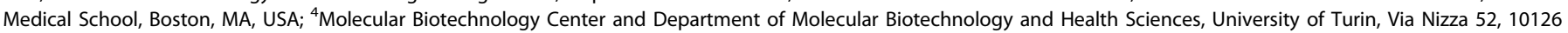

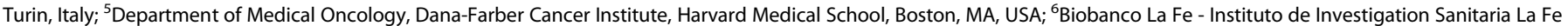

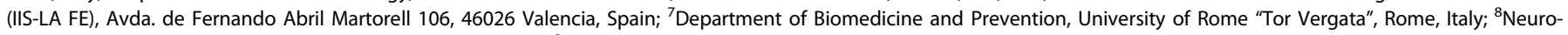

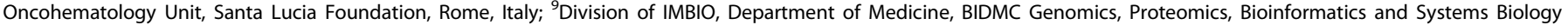

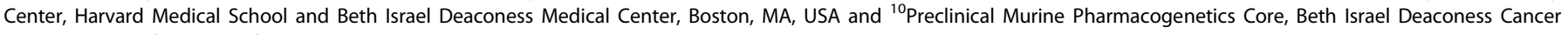
Center, Dana Farber/Harvard Cancer Center, Boston, USA

Correspondence: Pier Paolo Pandolfi (ppandolf@bidmc.harvard.edu)

These authors contributed equally: Riccardo Panella, Giulia Cheloni, Ming Chen

Received: 16 August 2018 Accepted: 12 March 2019

Published online: 25 April 2019 
in the treatment of AML harboring mutation of IDH in both an in vitro and in vivo setting.

\section{RESULTS}

Development of cell autonomous resistance to $\mathrm{mIDH} 2$ targeting by serial transplantation of mouse AML

Our previous modelling efforts have demonstrated that murine HSCs primed with doxycycline (DOX) inducible mIDH2 (IDH2 ${ }^{\mathrm{R} 140 \mathrm{Q}}$ ) expression and subsequently transduced with Hoxa9-GFP and Meis1a-IRES-YFP oncogenes give rise to AML that requires maintained expression of $\mathrm{mIDH} 2$ (DOX 'ON') to sustain the leukaemia. This dependence is demonstrated by collapse of the disease upon de-induction of $\mathrm{mIDH} 2$ (DOX 'OFF') in a primary or secondary transplant setting. ${ }^{13}$ However, our studies now show that in a third serial transplantation, this AML evolves to become independent from the oncogenic driver (Fig. 1a), and demonstrated a notable increase in disease burden (Fig.1b-e and Supplementary Information, Fig. S1a-f). This independence from $\mathrm{mIDH}$ expression may represent a suitable model for patients that present with de novo resistance to Enasidenib as reported in a recent clinical trial. ${ }^{8}$

Indeed, 2-HG levels observed in third transplant mice were as high as those in second transplant mice Supplementary Information, Fig. S1g), and while inactivation of the oncogene strongly reduced 2-HG levels, this failed to impact progression of the disease (Fig. 1f-i).

To further evaluate if the independence from $\mathrm{mIDH} 2$ in vivo represents a cell autonomous process, we established in vitro culture systems to assay stemness and the proliferative potential of these AMLs (Supplementary Information, Fig. S1h, i). In these conditions, AML cells from both second and third recipient mice demonstrated a similar capacity to generate colonies in methylcellulose (Fig. 1 j, vehicle treated). Targeted inhibition of $\mathrm{mIDH} 2$ activity in vitro decreased the colony forming capacity of AML cells isolated from second recipients, but not of those cells isolated from third recipient (Fig. 1j, k), though 2-HG levels were reduced (Fig. 1I), confirming the ability of our model system to mimic de novo resistance observed in the clinic as suggested above. All together these data demonstrate that while $\mathrm{mIDH} 2$ as an early event is required to sustain $\mathrm{AML}$, the disease evolves a cell autonomous independence from the $\mathrm{mIDH} 2$ oncogene. Serial transplantation in our mouse model mimics progression of the disease over time and offers the opportunity to test for dependence on mutant IDH alleles. Thus, we sought to study these secondary $\mathrm{mIDH} 2$-dependent and tertiary $\mathrm{mIDH} 2$ independent leukaemias to identify common and shared vulnerabilities that could be harnessed for therapy, and facilitate the use of mIDH-targeting agents in the clinic. As well, we wanted to profile $\mathrm{mIDH} 2$ independent leukaemia to predict for possible mutational landscapes associated with de novo resistance in human $\mathrm{mIDH}$ AML patients.

Evolution of AML harbouring $\mathrm{mIDH}$ is associated with a distinct metabolic switch to one-carbon metabolism and altered redox balance

Since the expression of $\mathrm{mIDH} 2$ directly impacts cellular metabolism, polar metabolites were extracted from sensitive and resistant AML cells from both second and third transplanted mice (Supplementary Information, Fig. S2a). Comparison of metabolite abundance established multiple metabolic processes altered in progression of the disease (Fig. 2a, b). Leukaemia cells derived from the third recipients showed significant enrichment in glycerophospholipid, pyrimidine, purine, cysteine and methionine metabolism pathways (Fig. 2b). These pathways constitute onecarbon metabolism network that includes both the folate and the methionine cycles (Supplementary Information, Fig. S2b), key metabolites in which showed increased levels in evolving AML
(Supplementary Information, Fig. S2c). Importantly, one-carbon metabolism is a main cellular engine contributing to nucleotide metabolism and global methylation, as well as cellular redox status. ${ }^{14}$ Similar to recent findings for purine metabolism in cancer, ${ }^{15}$ we observed a general increase in purine precursors in third compared to second recipient AMLs (Supplementary Information, Fig. S2d-f).

Finally, one-carbon metabolism also contributes to redox balance through the biosynthesis of glutathione (GSH). ${ }^{16}$ Glutathione metabolism was the most significant and highly enriched metabolic pathway in resistant AML cells (Fig. 2c). Third recipient AML cells demonstrated high levels of glutathione and cysteine, glutathione's major precursor (Fig. 2d), suggesting that these cells may have an altered redox balance. Indeed, altered NADH, NAD + and NADPH, NADP+ levels (Fig. 2e) and corresponding ratios (Supplementary Information, Fig. S2g) confirm this oxidative stress, and excessive ROS levels were visualized using MitoSOX ${ }^{\mathrm{TM}}$ Red staining (Fig. 2f) and demonstrated a significant increase in ROS in third recipient mice compared with second recipients (Fig. 2g).

Interestingly, analysis of early stage mIDH2 leukaemia in comparison with Hoxa9/Meis1a-harbouring wild type IDH alleles revealed that some of the metabolic features exacerbated during evolution were already present at this initial stage. Indeed, $\mathrm{mIDH} 2$ leukaemia at early stage, isolated from first recipients, demonstrate increased nucleotide metabolism (Supplementary Information, Fig. $\mathrm{S} 2 \mathrm{~h}-\mathrm{j}$ ) and upregulation of the glutathione pathway (Supplementary Information, Fig. S2k) as well as increased ROS levels (Supplementary Information, Fig. S2I).

These data suggest a metastable metabolic state where an altered redox balance of $\mathrm{mIDH} 2$ leukaemia arising from increased ROS is counterbalanced by the increased antioxidant GSH to prevent cells from catastrophic collapse.

To evaluate the contribution of increased ROS during time for cell damage such as genotoxic stress, we stained for $\mathrm{\gamma}$-H2A.X on mouse bone marrow leukaemia cells (Fig. 2h). Similarly, staining for $\mathrm{Y}$-H2A.X was carried out on infiltrated spleens from both second and third transplanted mice (Supplementary Information, Fig. S3a). Evidence of increased DNA damage suggests that altered redox balance may represent a vulnerability within $\mathrm{mIDH} 2$ leukaemias. Such a vulnerability could be exploited using arsenic trioxide, a well-known ROS producing and GSH inactivating agent used in the treatment of APL. ${ }^{17}$

To further determine if such genotoxic stress may result in DNA damage, we carried out whole exome sequencing (WES) of a second recipient and three of its derived third recipients (daughter leukaemia recipients) (Supplementary Information, Fig. $S 3 b) . G>A: C>T$ and $A>G: T>C$ transitions of nonsynonymous base substitutions accounted for two-thirds of the single base substitutions (SBS) observed, as is frequently found in cancers without biased substitution (Supplementary Information, Fig. S3c). A variety of mutations were observed (Supplementary Information, Table S1) in both second and third recipient-derived leukaemia cells underscoring the genotoxic state, yet only Sp140 appeared to be commonly mutated across all samples (Supplementary Information, Fig. S3d). Each of these leukaemias harboured a unique point mutation in Sp140 $\left(S p 140^{Y 5 D}\right.$ in third_11; Sp 140 R94Q in third_08; Sp 140 ${ }^{C 203 G}$ in third_09) (Supplementary Information, Table S1). Although the role and function of Sp140 in hematologic disease is poorly understood, Sp140 has been shown to be both a component of the PML nuclear body, ${ }^{18}$ and an interaction partner for Pin $1 .{ }^{19}$ However, no obvious alteration to Sp140 or PML localization was observed for these mutant variants (data not shown). Additionally, we demonstrate that mutant Sp140 maintains its ability to interact with PIN1 (data not shown), in agreement with the fact that the observed Sp140 mutations are not within the reported PIN1 interaction loop. 
a
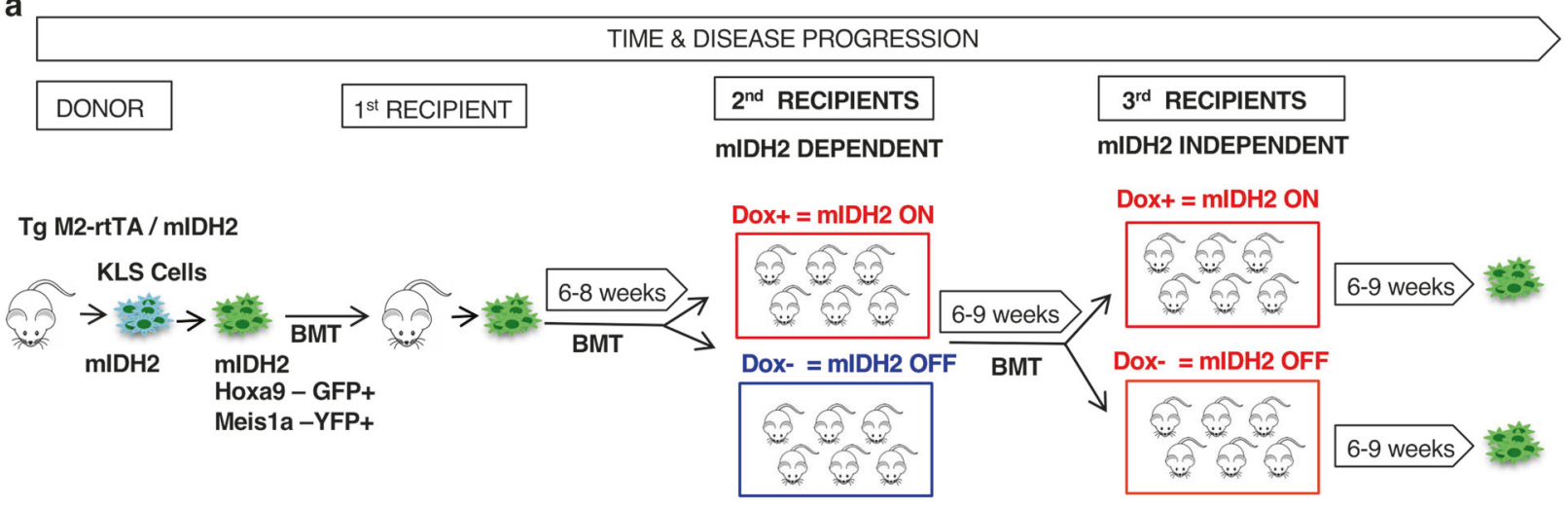

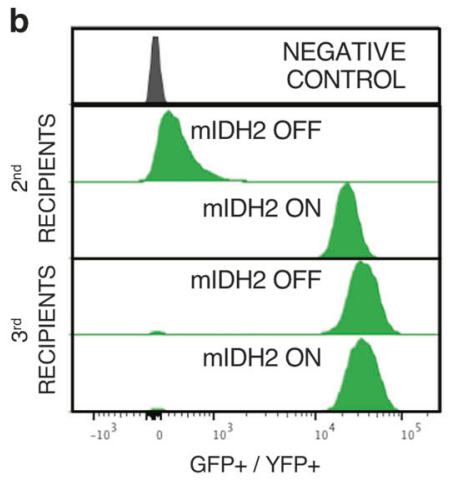

C

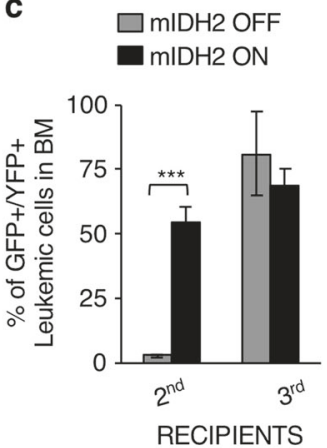

d

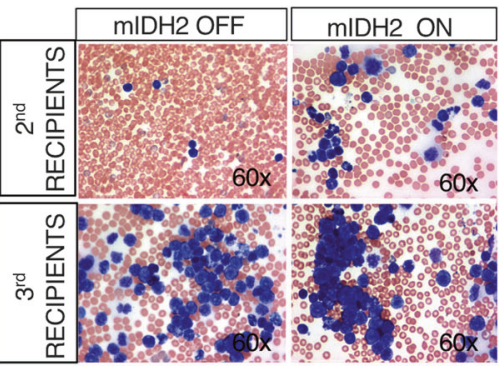

RECIPIENTS e $\square \mathrm{mIDH} 2 \mathrm{OFF}$

$\square \mathrm{mIDH} 2 \mathrm{ON}$

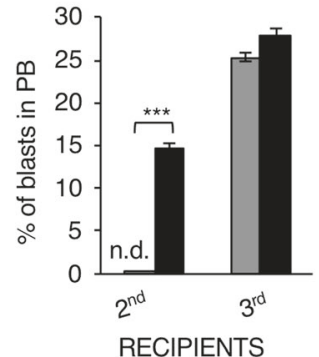

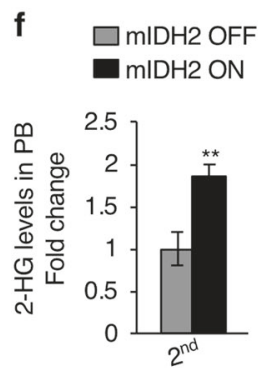

RECIPIENTS
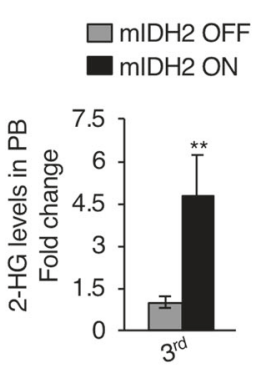

RECIPIENTS
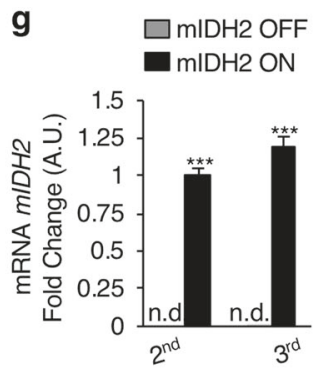

RECIPIENTS

h

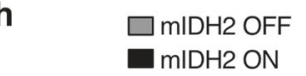

i

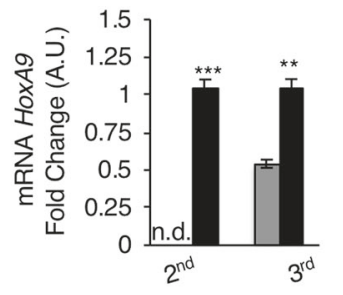

RECIPIENTS

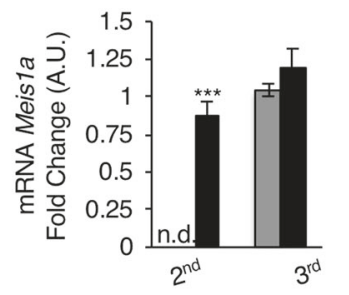

$\mathbf{k}$
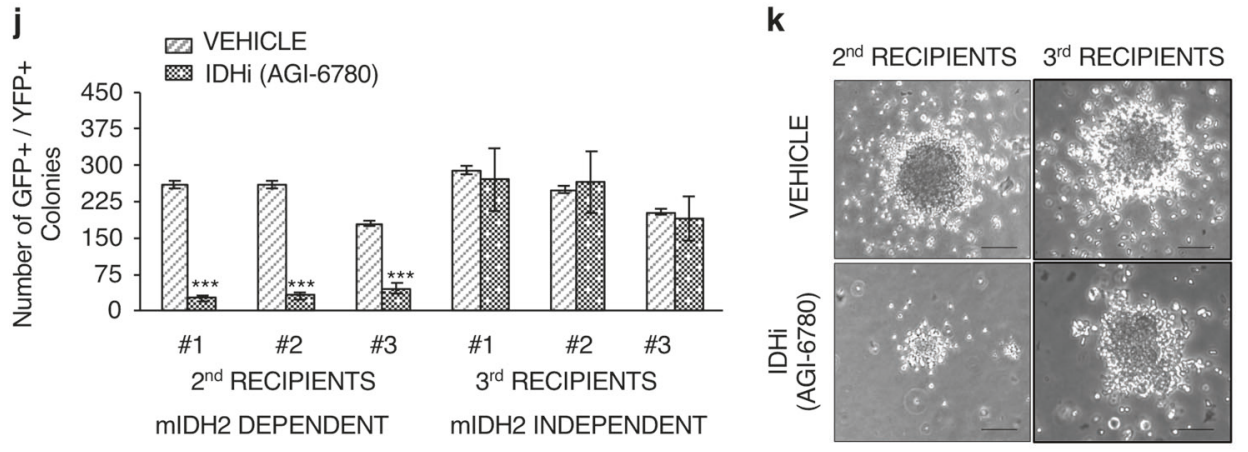

I

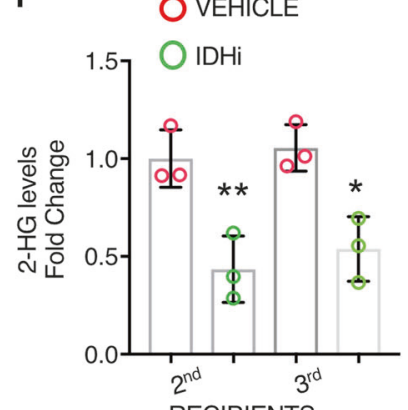

Global changes across these leukaemia genomes identified a distinct pattern of both genomic amplification and deletion in each AML (Supplementary Information, Fig. S3e). Alternations in several leukaemia-associated genes were found, including amplification of the Flt3 proto-oncogene (third_08) and deletion of the tumour suppressors Dok1 and Trp53 (third ${ }^{\text {rd }}$ 09) and Dok3 and Dok4 (third_11) (Supplementary Information, Fig. S3e). Interestingly, a number of chromosomal translocations shared amongst third recipient leukaemias may be particularly relevant in the context of this disease. These translocations were observed for hematopoietic genes including Rae1 (Rae1;Pappa; $\mathrm{t}(4 ; 2))$, Meis1 (Eea1:Meis1; t $(11 ; 10)$ ), and both Ppp1r13b (Aspp1) and Cadps2 (Ppp1r13b:Cadps2; $\mathrm{t}(6 ; 12)$ ) (Supplementary Information, Fig. 3e and Supplementary Information, Table S2).

Together these data indicate that although multiple and diverse genomic alterations are acquired during evolution, a unique target responsible for independence to the mutant IDH does not appear to be present. 
Fig. 1 Development and characterization of an in vivo approach to model independence from IDH2 $2^{\mathrm{R} 140 \mathrm{Q}}$ in $\mathrm{AML}$. a Approach used to model in vivo independence from $\mathrm{mIDH} 2\left(\mathrm{IDH} 2^{\mathrm{R} 140 \mathrm{Q}}\right.$ ) in $\mathrm{AML}$. Briefly, this model is based on our previously published model for $\mathrm{mIDH} 2 \mathrm{AML}$ using HOXA9/MEIS1a overexpression by Kats et. al., 2014. KSL cells inducibly overexpressing mIDH2 (by Doxycycline (DOX)) are transduced with retrovirus to overexpress the leukaemia oncogenes HOXA9 and MEIS1a and primary leukaemia allowed to establish in a primary host (first Recipient). Subsequenly, leukaemia cells are harvested and transplanted into a secondary host (second Recipient), at which point the disease remains dependent on $\mathrm{mIDH} 2$ expression as previoulsy published. Finally, leukaemia cells from the secondary host are further transplanted into a third host (third Recipient), at which point AML independent of $\mathrm{mIDH} 2$ arises and serves as a model for resistant disease. Red boxes: leukaemia state. Blue boxes: healthy state. b Representative flow cytometry plots of leukaemia bone marrow samples. $X$-axis, fluorescence Intensity (0-105), Y-axis, cell counts. c Percentage of GFP+/YFP+(blasts) quantitated by flow cytometry. d Representative May-GrünwaldGiemsa staining of peripheral blood (PB) smears at euthanasia of indicated models at 6-9 weeks after transplant. e Percentage of blasts in PB for the indicated models. f LC- MS quantification of the 2-HG in peripheral blood (PB). Data are mean \pm SD of $n=4$ samples, $t$-test; ** $p \leq 0.01$. g-i Quantitative PCR analyses for IDH2 ${ }^{\mathrm{R} 140 \mathrm{Q}}$, HoxA9 and Meis1a in BM blasts. Data are mean $\pm \mathrm{SD}$, one-way anova; ${ }^{* *} p \leq 0.01,{ }^{* * *} p \leq 0.001$. $\mathbf{j}$ Numbers of methylcellulose colonies generated at third plating by leukaemia cells (GFP + /YFP + ) isolated from $n=3$ bone marrow samples for each of the indicated models. IDH2 ${ }^{\mathrm{RT} 40 \mathrm{Q}}$ inhibitor (IDHi): AGI-6780, $1 \mu \mathrm{M}$, vehicle: DMSO. Data are means $\pm \mathrm{SD}$ of $n=3$ replicates for each sample; $t$-test; ${ }^{* * *} p \leq 0.001$. k Representative examples of methylcellulose colonies generated by blasts from the indicated AML models at third plating. I Relative LC- MS quantification of 2-HG from colonies pooled and extracted for metabolites at third-plating from the indicated models. $n=3$ samples. Data are mean $\pm \mathrm{SD}$, one-way anova; ${ }^{*} p \leq 0.05,{ }^{* *} p \leq 0.01$. BMT bone marrow transplant, BM bone marrow. Doxycycline supplemented diet: Dox+: mIDH2 ON,IDH2 ${ }^{\mathrm{R} 140 \mathrm{Q}}$ ON; Dox-: mIDH2 OFF; IDH2 ${ }^{\mathrm{R} 140 \mathrm{Q}}$ OFF. See also Supplementary Information, Fig. S1

Transcriptional rewiring characterizes evolution of $\mathrm{mIDH} 2$ leukaemia and primes for ATRA sensitivity

We next carried out RNAseq analysis to create a global picture of leukaemia progression from a state of $\mathrm{mIDH} 2$ dependence to independence (Supplementary Information, Fig. S4a), with primary leukaemias derived from Hoxa9;Meis1 overexpression without $\mathrm{mIDH} 2$ used for comparison. PCA analysis identified distinct clusters of second and third recipient leukaemias (Supplementary Information, Fig. S4b). Differential gene expression patterns highlighted clear differences between transplanted leukaemias (Supplementary Information, Fig. S4c). A gene-centric PCA identified Jun/Fos AP-1 transcription factor genes (Jun, Fos, Fosb) as well as genes associated with granulopoiesis, myeloid differentiation (Mpo, Lyz2, Ngp), ${ }^{20,21}$ and inflammation $(Z f p 36)^{22}$ as differentially expressed gene signatures (Supplementary Information, Fig. S4d).

Furthermore, significant upregulation of cancer-associated signalling pathways including MAPK, Erk, PI3K and Akt was observed in third transplanted leukaemias, as well as pathways associated with inflammation and tumour microenvironment (IL-1, IL-6, ILK, MIF, TNFR2, etc.) (Supplementary Information, Fig. S4e). Gene set enrichment analysis (GSEA) identified similar pathways altered in resistant AMLs, including MAPK, TNF signalling, and pathways related to Tretinoin response (aka. all-trans retinoic acid; ATRA) (Fig. 3a, b), while an interactome network of all differentially regulated genes highlighted the central role of Erk/MAPK signalling in defining the signatures associated with resistant disease (Supplementary Information, Fig. S4f, g). Enrichment of these signatures was also confirmed in primary (first transplant) $\mathrm{mIDH} 2 \mathrm{AML}$, where MAPK signalling and PI3K/Akt signalling pathways were enriched compared with HoxA9/Meis1a leukaemias (Supplementary Information, Fig. S4h). In addition, GSEA demonstrated sensitivity to Tretinoin in primary (first transplant) mIDH2 AML (Supplementary Information, Fig. S4i, j). Collectively these data suggest that such aberrant transcriptional signatures are present at the time of leukaemia initiation, and become ever more dominant as the leukaemia evolves. Indeed, our findings are in line with the recent reporting of mutations to MAPK and RAS signalling pathways in $\mathrm{mIDH} 2 \mathrm{AML}$ with acquired resistance to Enasideb in the clinic, ${ }^{8}$ and is also consistent with previously published data highlighting the major impact of MAPK signalling pathways in shaping the epigenome of FLT3-ITD AML. ${ }^{23}$

Given the striking and paradoxical observation regarding aberrant Tretinoin / ATRA response, and the fact that it has been previously demonstrated by Schenk et al. (2012) that inhibition of the LSD1 histone demethylase in human AML cell lines (TEX and $\mathrm{HL}-60$ ) is associated with an increased sensitivity to ATRA, we next ascertained whether LSD1 activity is impaired in $\mathrm{mIDH} 2 \mathrm{AML}$. Data from Schenk et al. demonstrate a distinct gene signature associated with ATRA treatment in the context of LSD1 inhibition (See Schenk et al. ${ }^{24}$. Supplementary Data 1; TEX + HL-60: ATRA + TCP vs. untreated $)^{24}$ and by comparing this set of ATRA responsive genes identified by Schenk et al. ${ }^{24}$ (Fig.3c, Venn diagram, Group B, human gene set) with the set of the upregulated genes in our mouse mIDH2 AML (Fig. 3c, Venn diagram, Group A, mouse gene set), we identified a significant overlap of 48 genes that were upregulated in both. These data were suggestive of a transcriptional state resembling one associated with ATRA sensitivity upon LSD1 inhibition, and suggest that LSD1 may be deactivated in some manner. ${ }^{23,24}$ Interestingly, these 48 shared genes were found to be upregulated in both second and third transplanted AMLs relative to a non-mIDH2 control AML (Fig. 3d), again suggesting that both early and late stage $\mathrm{mIDH} 2$ AMLs might be primed for response to ATRA through inhibition of LSD1 (Supplementary Information, Fig. S4k, I). Our findings therefore seemed paradoxical and at odds with the fact that $\mathrm{mIDH} 1 / 2$ are known to block myeloid differentiation. This in turn led us to analyse in greater detail ATRA pathways in the progression of mIDH2 AML. GSEA analysis highlighting Tretinoin signalling suggested that $\mathrm{mIDH} 2$ leukaemias isolated from third recipients are characterized by a gene expression profile diametrically opposed to an ATRA response, ${ }^{25}$ with expression of genes repressed by treatment with ATRA (Tretinoin Down Signature; Down-regulation of Tretinoin genes), and depletion of genes activated by treatment with ATRA (Tretinoin Up Signature; Upregulation of Tretinoin genes) (Fig. $3 a, b$ and Supplementary Information, Table S3). In turn, these data suggested that AML derived from third recipients experience a profound alteration of the ATRA pathway, and prompted us to hypothesize that $\mathrm{mIDH} 2$ AML may be sensitized to ATRA, in line with previous work examining ATRA sensitivity in the context of mutant IDH1. ${ }^{26}$ Indeed, although the Tretinoin GSEA data would suggest that the leukaemia is attempting to drift away from sensitivity to ATRA, the inhibition of LSD1 might actually perhaps maintain the ATRA sensitivity and this vulnerability as the leukaemia progresses.

To better understand if LSD1 activity is indeed compromised upon $\mathrm{mIDH}$ overexpression and to evaluate if LSD1 target genes were more susceptible to activation by ATRA in this setting, we generated TF1 and U937 cell lines overexpressing the $\mathrm{mIDH} 2$ allele with increased 2-HG levels (Supplementary Information, Fig. S5a, b). TF1 cells have been extensively utilized to study $\mathrm{mIDH}$ function, where its expression results in a block of cellular differentiation. ${ }^{27}$ Surprisingly, we detected a striking lack of LSD1 activity in extracts isolated from $\mathrm{mIDH} 2$ cells (Fig. 3e, f). By evaluating the expression of ITGAM, LILRA5, RARA and PRAM1, direct targets of LSD1, which were reported to be upregulated in 
a

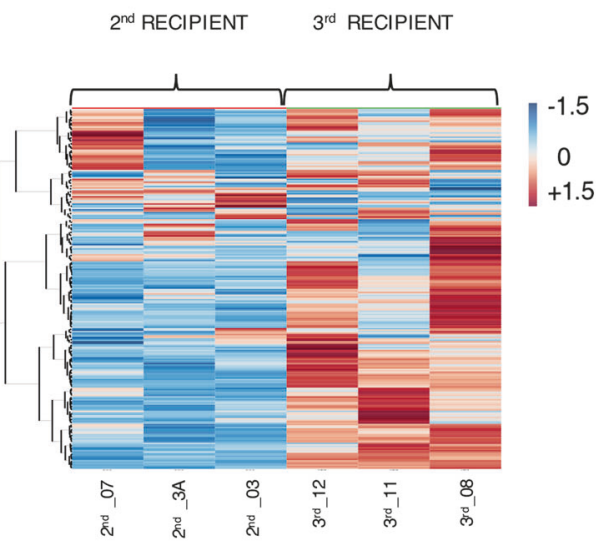

C

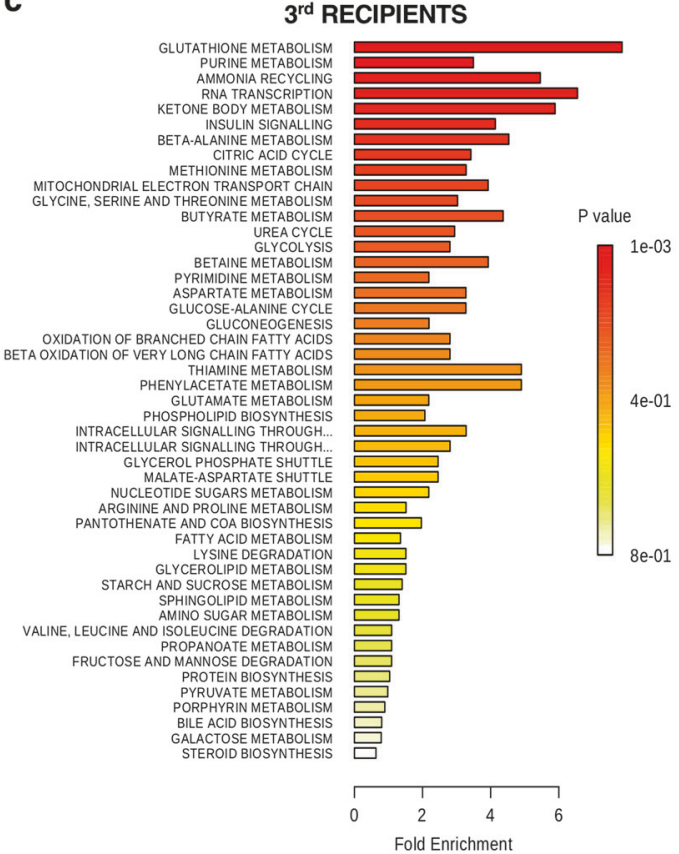

d

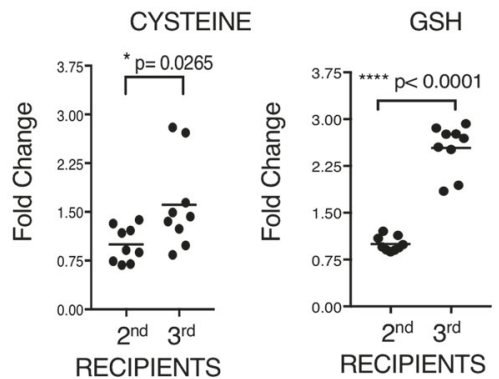

b

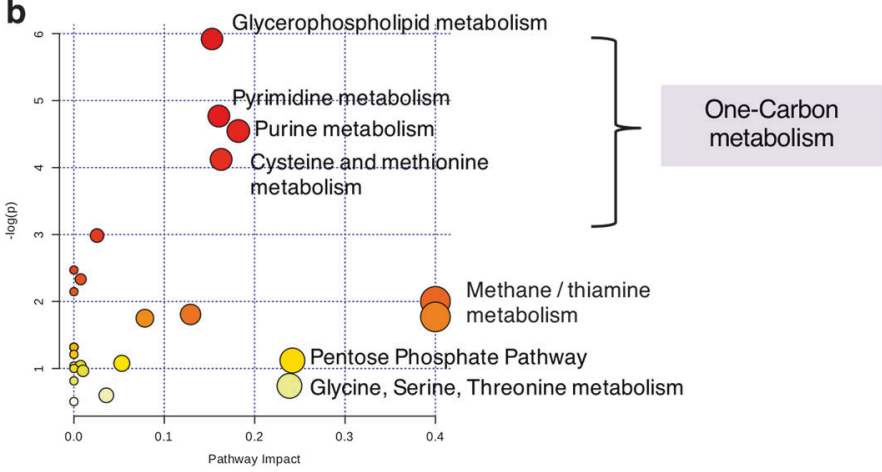

e
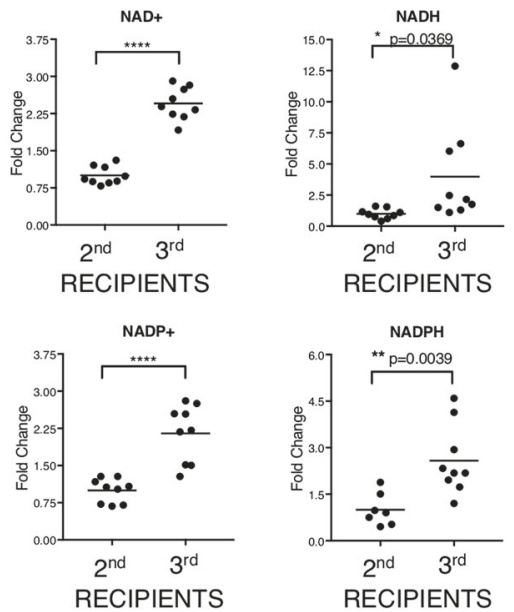

f MITOCHONDRIAL REDOX STATE

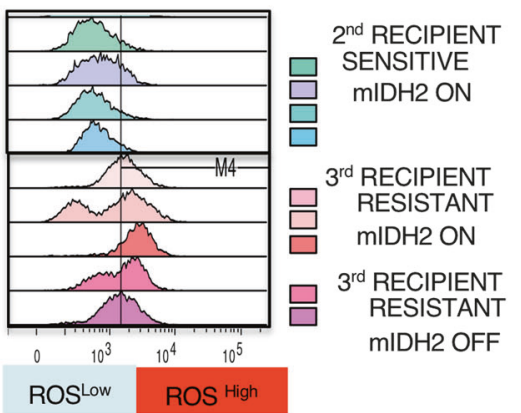

g

- $\mathrm{mIDH} 2 \mathrm{ON}$ mIDH2 OFF

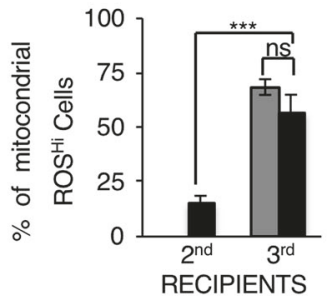

h
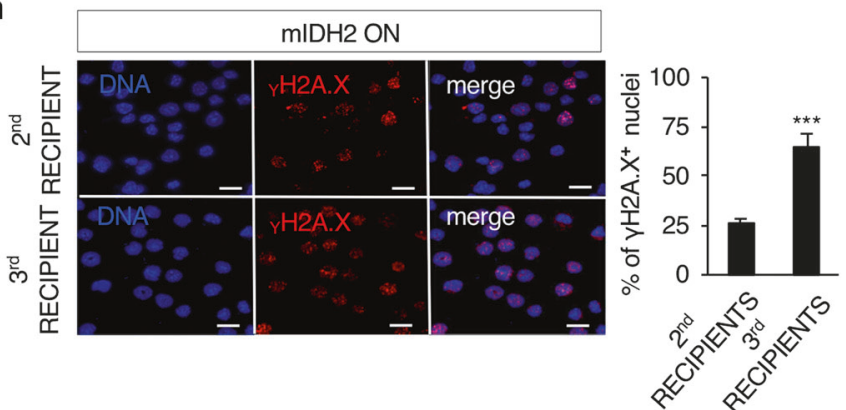

leukaemia cells by treatment with ATRA in the context of LSD1 inhibition (see Schenk et al. ${ }^{24}$ 2012. Supplementary Data 1; TEX + HL-60: ATRA + TCP vs. untreated), we show that mutant IDH2 in combination with ATRA treatment mimics the previously reported inhibition of LSD1 in combination with ATRA treatment in human leukaemia cell lines (TCP + ATRA treatment in TEX, HL-60 leukaemia cells) resulting in a significant induction of these genes (Fig. 3g). Similarly, TF-1 cells overexpressing mIDH1 (Supplementary Information, Fig. S5c, d) demonstrated a comparable susceptibility of these LSD1 gene targets to activation upon 
Fig. 2 Leukaemia progression to $\mathrm{mIDH} 2$ independence is marked by switching to One-Carbon metabolism and increased genotoxic stress. a Hierarchical clustering of leukaemia cells' metabolites isolated from Dox + cohorts. Rows: metabolites; columns: samples; colour key indicates metabolite abundance level (blue: lowest; red: highest). Clustering generated by MetaboAnalyst's annotation tool. b Pathway enrichment analysis shows altered metabolic pathways between secondand third recipient derived leukaemia cells. c Bar chart showing metabolic pathways enriched in leukaemia cells isolated from third recipients. d LC-MS/MS fold change levels of Cysteine (CYS) and Glutathione (GSH) metabolites in leukaemia cells isolated from second or third recipients. Data are mean \pm SD; of at least $n=3$ different samples; unpaired $t$-test; ${ }^{*} p \leq 0.05,{ }^{* * * *} p \leq 0.0001$. e LC-MS/MS fold change levels of NAD+/NADH and NADP+/NADPH in leukaemia cells isolated from second or third recipients. NAD+ nicotinamide adenine dinucleotide oxidized, NADP+ nicotinamide adenine dinucleotide phosphate oxidized, NADH nicotinamide adenine dinucleotide reduced, NADPH nicotinamide adenine dinucleotide phosphate reduced. Data are mean \pm SD; unpaired $t$ test; ${ }^{*} p \leq 0.05,{ }^{* *} p \leq 0.01,{ }^{* * *} p \leq 0.0001$. f Representative Flow cytometry plots showing fluorescence intensity of leukaemia cells isolated from second $(n=4)$ or third $(n=5)$ recipients and incubated with the mitochondrial superoxide indicator, MitoSox. $X$-axis: Fluorescence intensity levels; $Y$-axis: relative cell counts. g Quantitation of mitochondrial ROS (ROS ${ }^{\mathrm{Hi}}$ ) in leukaemia cells derived from second or third RECIPIENT AML samples $\left(n=2\right.$ DOX, $\left.n=3 \mathrm{DOX}^{+}\right)$cells as shown in f. Data are mean \pm SD; one-way anova; ns not significant, ***p $\leq 0.001$. $\mathbf{h}$ Immunofluorescence for the DNA damage marker $\gamma \mathrm{H} 2 \mathrm{AX}$ (red) and DNA (blue) and relative quantitation of positive nuclei. Data are mean $\pm \mathrm{SD}$; unpaired $t$-test; ${ }^{* * *} p \leq 0.001$. Dox $+\operatorname{mIDH} 2$ ON,IDH2 ${ }^{\mathrm{R} 140 \mathrm{Q}} \mathrm{ON}, \mathrm{Dox}+\mathrm{mIDH}_{2} \mathrm{OFF}, \mathrm{IDH} 2^{\mathrm{R} 140 \mathrm{Q}}$ OFF. n.d. not detected. See also Supplementary Information, Figs. S2-S3

treatment with ATRA (Supplementary Information, Fig. S5e). In addition, we confirmed that the expression of the differentiation markers CD71 and CD235a induced in TF1 cells overexpressing $\mathrm{mIDH}$ by treatment with ATRA resembles their expression on normal TF1 cells treated with the combination of ATRA and LSD1 inhibitor. Furthermore, combination of LSD1 inhibition and treatment with ATRA induces higher expression of CD71 and $\mathrm{CD} 235 \mathrm{a}$ in $\mathrm{mIDH} 2$ cells (Supplementary Information, Fig. S5f).

On this basis, we further examined the mechanism by which mIDH2 confers sensitivity to ATRA while at the same time blocking differentiation. We hypothesized that the leukemic cells would be in a metastable state - sensitized to ATRA on the one hand, yet with the ATRA signaling pathway suppressed on the other. While LSD1 inhibition can facilitate sensitivity to ATRA, we reasoned that the PIN1 proto-oncogene could be at the center of this paradox of simultaneous suppression, as it is an established negative regulator of the ATRA nuclear receptor pathway. ${ }^{28}$ Indeed, we previously demonstrated that the transcriptional response of genetic inactivation of PIN1 faithfully mimics that of ATRA treatment in the APL cell line NB4 ${ }^{29}$ and that, critically, ATRA is a direct inhibitor of Pin $1 .{ }^{29}$ We therefore hypothesized that while PIN1 may contribute to proliferative signalling (MAPK- and PI3K-dependent) and increased survival to ROS-mediated apoptosis, its overexpression may also drive the block of differentiation and trigger the "ATRA-off" gene signature observed in $\mathrm{mIDH} 2$ evolving AML (Fig. $3 a, b$ ). Conversely, pharmacological doses of ATRA can function to inhibit PIN1, which might lead to the prediction of an exquisite sensitivity of mIDH leukaemia to ATRA.

In line with this hypothesis, we confirmed a marked upregulation of Pin 1 protein in $3^{\text {rd }}$ transplanted murine AML in both the presence and absence of $\mathrm{mIDH} 2$ (Fig. 3h, Lanes $5-8$ ). We next examined the status of retinoic acid receptor (RAR) genes in the evolving AML and observed strong downregulation of both Rara and Rxra in third recipient AML (Fig. 3h, Lanes 5-8 compared with Lanes 1 and 2), in line with Pin 1 degradation of Rara. ${ }^{30}$ On the other hand, downregulation of the tumour suppressive p42 form of C/EBPa, and decreased expression of C/EPBE were observed, which may also contribute to the evolving aggressiveness of the disease mediated by PIN1 ${ }^{31}$ (Fig. 3 h).

To further support the role of PIN1 as such a critical contributor to $\mathrm{mIDH} A M L$, we treated cells overexpressing PIN1 in vitro with ATRA, and demonstrated that this promotes expression of the differentiation marker CD71. Importantly, differentiation was further potentiated by combining ATRA with the LSD1 inhibitor, GSK2879552 (Supplementary Information, Fig. S5g). Analogously, we confirmed that cells overexpressing PIN1 are sensitized to ATO treatment while LSD1 inhibition appears to play no role in exacerbating this phenotype (Supplementary Information, Fig. S5h).
We next utilized our mIDH2 overexpressing TF-1 and U937 cell lines to understand if the sensitivity to ATO and ATRA was recapitulated in human leukaemia. Indeed, both TF-1 (Fig. 3i) and U937 (Fig. 3j) lines demonstrated upregulation of PIN1 protein in the presence of $\mathrm{mIDH} 2$ (Fig. $3 \mathrm{i}, \mathrm{j}$ ), as well as retinoic acid receptors and C/EBPa. This was further supported by increased PIN1 mRNA levels in TF-1 and U937 cells overexpressing mIDH2 (Supplementary Information, Fig. S6a, b). Furthermore, this was also observed to be the case for TF-1 cells overexpressing mutant IDH1 (Supplementary Information, Fig. S6c). Importantly, comparison of a cohort of primary AML patients with or without $\mathrm{mIDH} 2$ mutation identified increased levels in PIN1 mRNA levels in patients with $\mathrm{mIDH} 2$ mutation (Supplementary Information, Fig. S6d). Although TCGA AML samples represent a cohort of primary AML patients at early diagnosis, concomitant analysis demonstrated variable PIN1 expression, and patients harbouring $\mathrm{mIDH}$ mutations had a trend towards higher PIN1 expression (Supplementary Information, Fig. S6e). Indeed, we observed a similar trend in PIN1 protein levels assessed by intracellular flow cytometry from primary AML patients (Supplementary Information, Fig. S6f).

Therapeutic vulnerabilities driven by $\mathrm{mIDH}$

Given these findings we hypothesized that at the time of leukaemia initiation in mIDH primed cells, molecular, metabolic and transcriptional features promote PIN1 upregulation, transcriptional rewiring of ATRA signaling, and high ROS levels, which represent therapeutic vulnerabilities that can be harnessed for intervention in mIDH leukaemia.

As we recently reported that pharmacological doses of ATRA can inhibit the enzymatic activity of its negative regulator PIN1 through direct binding, ${ }^{29}$ and we here identified the loss of LSD1 activity in mIDH2 AML, we hypothesised that ATRA utilization can impact mIDH2 leukaemias at two levels (Fig. 4a). To test this, we first evaluated the ability of ATRA to promote differentiation of leukemic blasts in our murine model of AML. Indeed, both pharmacological and sub-pharmacological doses of ATRA demonstrated striking and increased differentiation effects on murine $\mathrm{mIDH} 2$ expressing AMLs as the disease evolves (Supplementary Information, Fig. S6g). To further determine whether the sensitivity to ATRA is indeed mIDH2-dependent and whether this would also be the case in human hematopoietic cells expressing $\mathrm{mIDH} 2$, we utilized our TF1 cell lines overexpressing the $\mathrm{mIDH} 2$ allele. Treatment of $\mathrm{mIDH} 2$ overexpressing TF1 cells with a pharmacological ATRA dose $\left(10^{-6} \mathrm{M}\right)$ resulted in a dramatic difference in cellular differentiation as compared to controls (Fig. 4b, c), with a concomitant decrease in cell growth (Supplementary Information, Fig. S6h). Furthermore, we generated U937 cells overexpressing the mutant isoform R140Q of IDH2 (Fig. 3j). This cell line has been extensively utilized to study how the fusion genes of APL (e.g. 

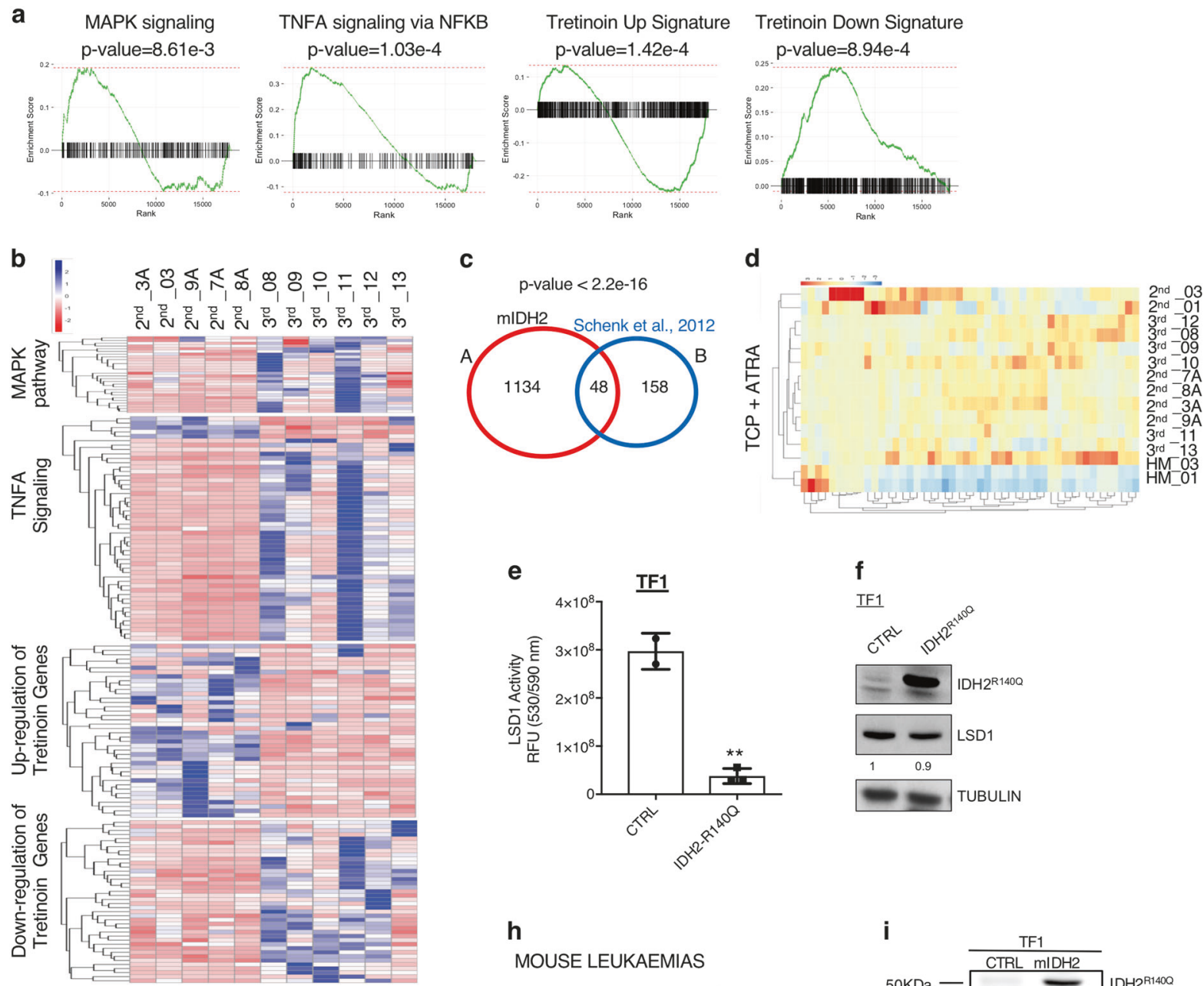

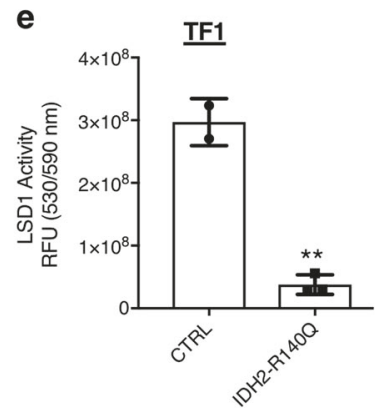

h

\section{MOUSE LEUKAEMIAS}
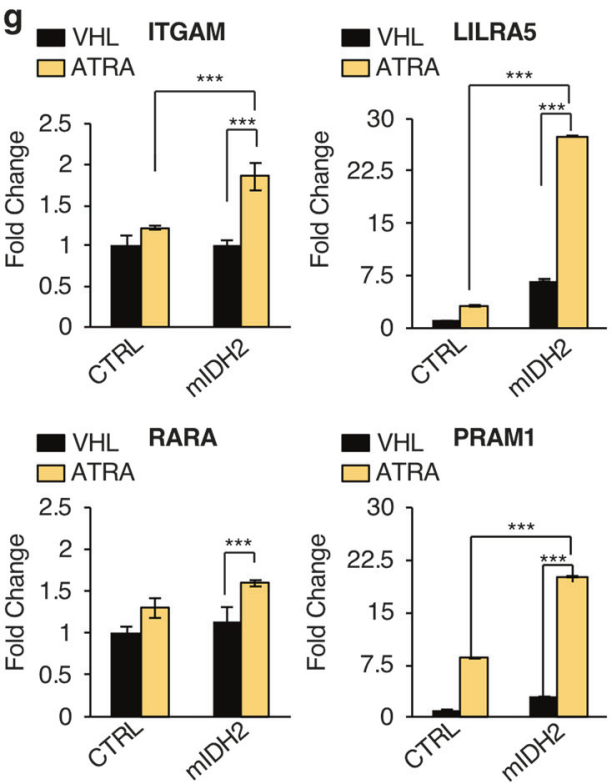

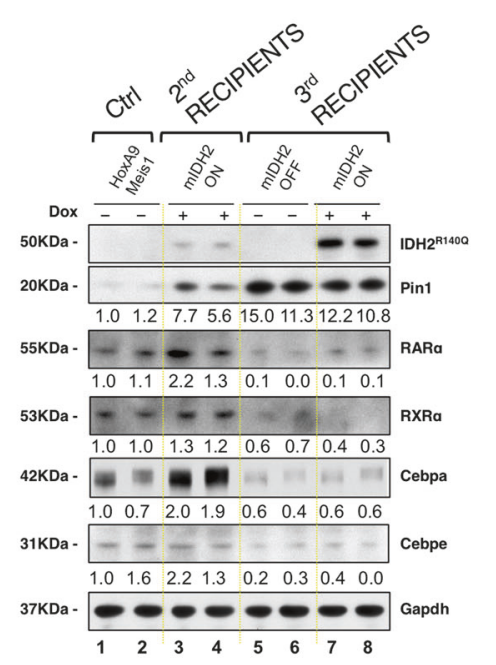

i
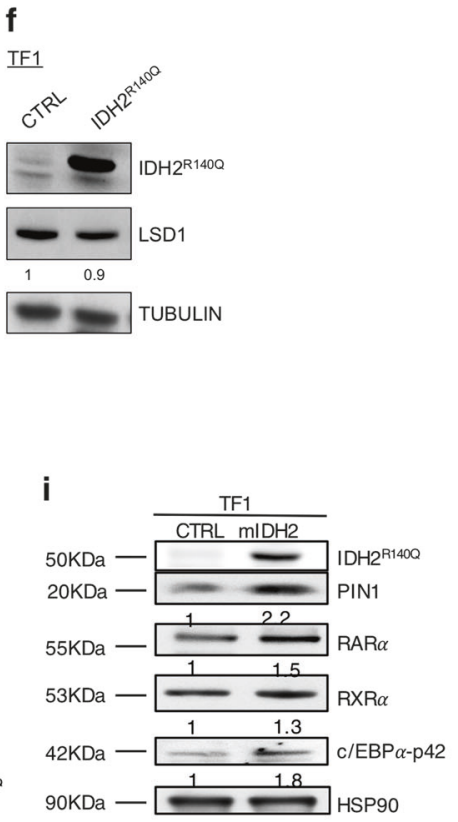

j

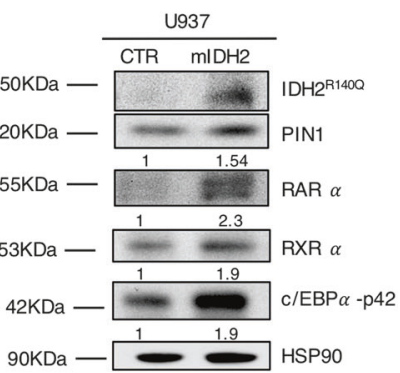

PML-RARa) cause a block in cellular differentiation. ${ }^{32,33}$ Once again, treatment of $\mathrm{mIDH} 2$-overexpressing U937 cells with pharmacological doses of ATRA $\left(10^{-6} \mathrm{M}\right)$ in the presence of vitamin $D$, resulted in a dramatic difference in cellular differentiation as compared to controls (Supplementary Information, Fig. S6i-
I) and a concomitant decrease in proliferation (Supplementary Information, Fig. $\mathrm{S} 6 \mathrm{~m}$ ), while treatment of our TF1 cell line overexpressing $\mathrm{mIDH} 1$ also demonstrated a similar decreased proliferative response (Supplementary Information, Fig. S6n). To determine if this sensitivity is dependent on PIN1 activity, we then 
Fig. 3 The transcriptional rewiring of $\mathrm{mIDH}$ leukaemia evolution is characterized by altered MAPK and ATRA associated pathways. a Gene Set Enrichment Analysis (GSEA) plots for MAPK, TNF $\alpha$, and ATRA related signatures in third recipient- vs second recipient- derived leukaemia cells. b Heat maps showing the relative abundance of genes specifically enriched in GSEA (a). c Venn diagram showing shared genes between mouse mIDH2 leukaemia and Schenken et al.; 2013 gene sets. d Heat map showing that shared genes shown in c are regulated concordantly in samples derived from second or third recipients with respect to ATRA + TCP (LSD1 inhibitor) gene sets reported by Schenken et al.; 2013. e Histogram showing LSD1 activity measured in nuclear extracts of mIDH2 TF1 (IDH2-R140Q) and relative controls. Data are mean \pm SD; of $n=$ 2 samples; unpaired $t$-test; ${ }^{* *} p \leq 0.01$. f Western blot assay on TF1 cells overexpressing the mutant R140Q of IDH2 (IDH2R140Q) or respective control cells (CTRL). LSD1 protein levels are slightly reduced in the mutant R140Q of IDH2 (IDH2R140Q). g Quantitative PCR analyses for genes associated with ATRA sensitivity in the context of LSD1 inhibition in human TF1 cells treated for $24 \mathrm{~h}$ with ATRA (1 $\mu \mathrm{M})$ or vehicle (DMSO). ITGAM integrin subunit alpha M, LILRA5 leukocyte immunoglobulin like receptor A5, RARA retinoic acid receptor alpha, PRAM1 PML-RARA regulated adaptor molecule 1. Data are mean $\pm S D$; one -way anova, ${ }^{* * *} p \leq 0.001$. $\mathbf{h}$ Western blot analysis for ATRA targets (Pin1), ATRA receptors (RAR $\alpha, R X R \alpha)$, and factors associated with ATRA-related differentiation pathway in hematopoietic cells (c/EBP $\alpha$, c/EBPE). The samples are protein extracts from independent $(n=2)$ leukaemia cells isolated from DOX+ (mIDH2 ON) or DOX (mIDH2 OFF) second or third recipients. Protein samples isolated from Hoxa9/Meis1a leukemic cells $(n=2)$ were included as control. $\mathbf{i}-\mathbf{j}$ Western blot analysis for factors associated with ATRA-related pathway in TF1 and U937 cell line, stably overexpressing the mutant isoform R140Q of IDH2 (mIDH2) or the empty vector (CTRL). mIDH2: IDH2 ${ }^{\mathrm{R} 146 \mathrm{Q}}$. See also Supplementary Information, Figs. S4 - S6 and Supplementary Information, Table S3

assessed whether PIN1 could act as a critical mediator of the block of differentiation observed in $\mathrm{mIDH} 2$ leukaemia through both pharmacological and genetic targeting. Inhibition of PIN1 by using a specific inhibitor (Juglone) clearly demonstrated Pin 1 to be differentially required in mouse AML cells harbouring $\mathrm{mIDH} 2$ which showed a significantly decreased colony forming capacity upon Pin1 targeting (Fig. 4d), while mIDH2 TF1 cells also demonstrated a similar sensitivity as demonstrated by reduced clonogenic capacity upon specific targeting of PIN1 by Juglone (Fig. 4e). Similarly, dependence on Pin 1 in $\mathrm{mIDH} 2$ independent $\mathrm{AML}$ cells was demonstrated by genetic silencing, which significantly attenuated colony forming capacity of these cells (Supplementary Information, Fig. S7a). Congruent with this behaviour, mIDH2- overexpression in TF1 cells inhibits the induction of the differentiation marker CD71 upon differentiation driven by erythropoietin (EPO), while specific targeting of PIN1 with Juglone restores the upregulation of this marker (Supplementary Information, Fig. S7b). These findings are mimicked by genetic targeting of PIN1 in mIDH2- overexpressing TF1 cells, with upregulation of the CD71 and CD44 markers detected by flow cytometry (Supplementary Information, Fig. S7c, d) and the differentiation associated $H G B$ and KLF1 genes demonstrating the increased sensitivity to differentiation upon loss of PIN1 (Supplementary Information, Fig. S7e, f). Critically, the overexpression of PIN1 favoured the maintenance of the distinctive undifferentiated state of $\mathrm{mIDH} 2$ cells, even upon treatment with the specific mutant IDH2 inhibitor AG-221, rendering them resistant (Supplementary Information, Fig. S7g-i), highlighting the dependence on Pin 1 and underscoring the relationship between $\mathrm{mIDH} 2$ and PIN1 (Supplementary Information, Fig. S7j-m).

In the same fashion, we evaluated the therapeutic opportunity afforded by ROS induction and concomitant PIN1 upregulation in this model through the use of arsenic trioxide (ATO), hypothesizing that the pro-oxidant activity of ATO could target Pin $1,34,35$ while increasing the intracellular ROS levels and leading cells to collapse and death (Fig. 4f). Indeed, our murine AML model demonstrated increased apoptosis in both a sensitive $\mathrm{mIDH} 2-$ dependent and resistant $\mathrm{mIDH} 2$-independent setting (Fig. 4g), while the expression of $\mathrm{mIDH} 2$ in TF1 cells conferred sensitivity to induction of apoptosis upon treatment with ATO (Fig. 4h). Interestingly, we also demonstrated the ability of $\mathrm{mIDH} 2$ and PIN1 overexpression to facilitate the induction of ROS in TF1 cells (Supplementary Information, Fig. S7n), while TF1 cells overexpressing $\mathrm{mIDH} 1$ were also found to respond to treatment with ATO (Supplementary Information, Fig. S7o).

Thus, we hypothesized that these agents may function as targeted therapies towards $\mathrm{mIDH}$ AML, especially as both ATRA and ATO have also been shown to be able to directly and indirectly target PIN1 at multiple levels consistent with our data. ${ }^{28,29,34-37}$ When these agents were combined to treat AML cells isolated from Hoxa9/Meis1a and Hoxa9/Meis1a/mIDH2 murine leukaemias in vitro, only mIDH2-expressing $\mathrm{AML}$ cells demonstrated enhanced sensitivity to the combination as measured by reduced colony forming capacity. Indeed, second transplanted AML demonstrated sensitivity to both single agents and a synergistic response to combination (Fig. 4i). Late stage disease (i.e. third recipient, $\mathrm{mIDH} 2$-independent leukaemias in both the presence and absence of $\mathrm{mIDH} 2$ ) demonstrated a similar pattern of responses, showing sensitivity to single agents and synergistic response to combination treatments (Fig. 4j). This indicates efficacy of these agents in both $\mathrm{mIDH} 2$ dependent and independent disease and is consistent with our hypothesis based on the AML RNAseq data (Fig. 3 and Supplementary Information, Fig. S5). Finally, to ensure that the efficacy conferred by treatment of $\mathrm{mIDH} 2 \mathrm{AML}$ with the ATRA/ATO combination was not due simply to oncogenic interaction with HoxA9 and Meis1a, we evaluated the ability of ATRA and ATO to effectively target mIDH AMLs derived from a number of distinct genetic contexts. In particular we analysed the clonogenic capacity of murine leukaemia cells derived from $\mathrm{AML}$ with $\mathrm{mIDH} 2$ in combination with FLT3-ITD, and mIDH1 in combination with NPMc+ or with FLT3-ITD/NPMc+(Fig. 4k). Each of the AMLs harbouring mIDH demonstrated exquisite sensitivity to the ATRA/ATO combination as compared to AML derived from MLL-AF9 overexpression with wild type IDH alleles, as negative controls. Murine AML arising from the combination of NPMc+/FLT3-ITD alone was used as a positive control as this genotype was previously shown to confer sensitivity to ATRA/ATO. ${ }^{38}$ However, mIDH AMLs conferred superior sensitivity even when compared with AML with this genetic make-up (Fig. 4k).

ATRA and ATO combination for treatment of mIDH AML

In order to evaluate the efficacy of this treatment in a human setting, we first carried out an in vivo pre-clinical trial using our U937 cell line in immunocompromised NSG host mice (Supplementary Information, Fig. S8a). Twenty-one days after initiating treatment, mice engrafted with $\mathrm{mIDH}$ 2-overexpressing U937 cells treated with the ATO/ATRA combination remained alive, while all control mice had succumbed to disease (Supplementary Information, Fig. S8b). Bone marrow (BM) analysis demonstrated a significant decrease in the percentage of human leukemic blasts, and increased differentiation, in both the ATRA and ATO/ATRA treated cohorts of mice transplanted with mIDH2-overexpressing cells (Supplementary Information, Fig. S8c-e). Importantly, only mIDH2-expressing U937 cells treated with the combination therapy demonstrated the mature myeloid CD11b marker amongst all the cohorts analysed (Supplementary Information, Fig. S8f, g). Reduced spleen weights were observed in mice transplanted with mIDH2-expressing U937 cells treated with ATO/ 
a

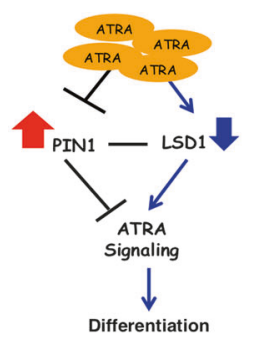

b
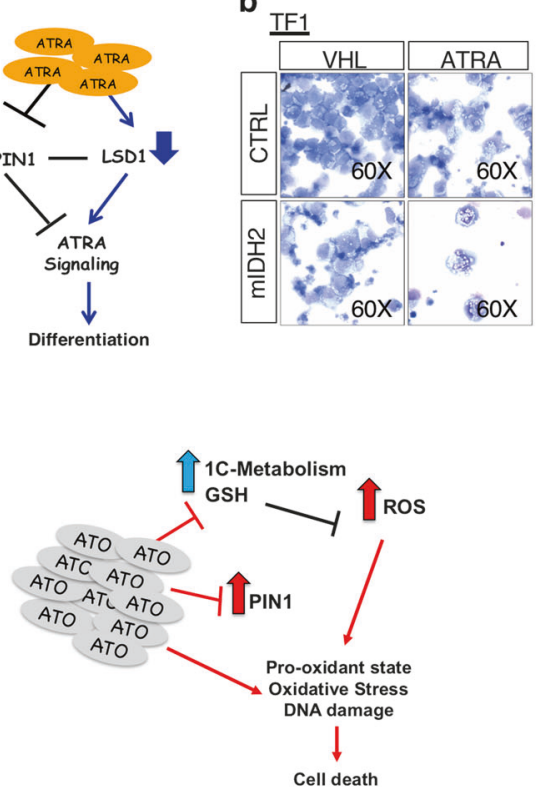

C

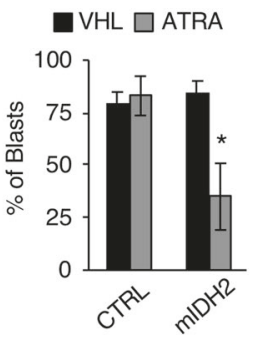

d

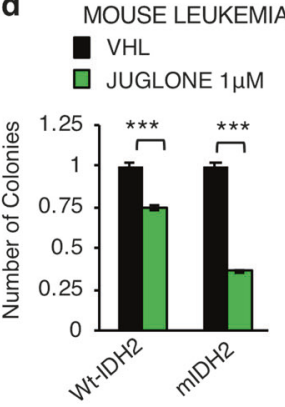

e

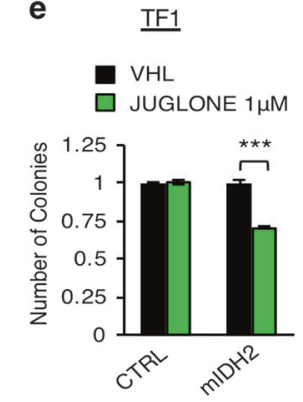

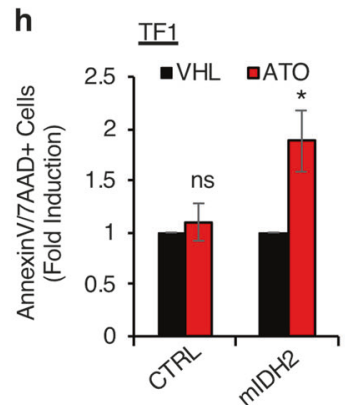

i
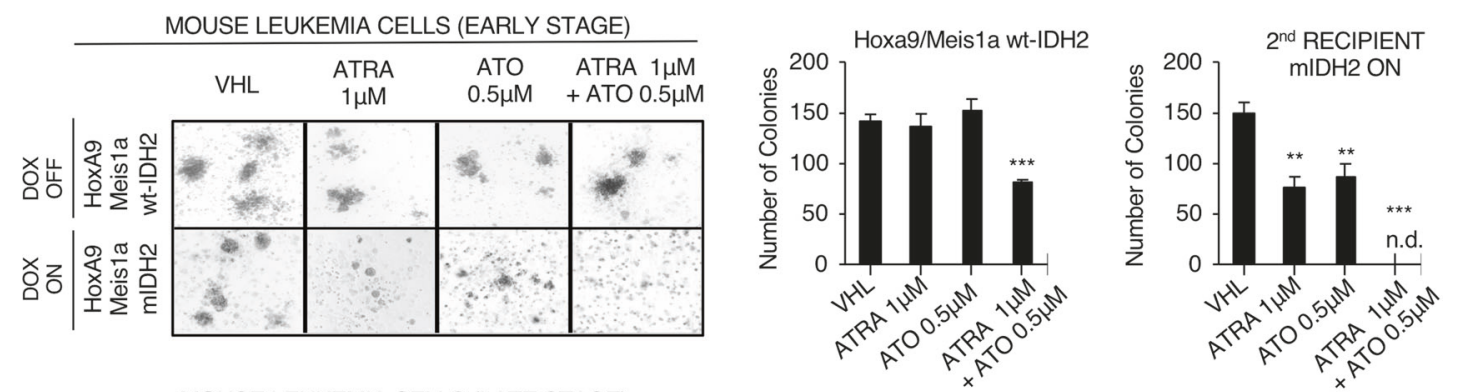

j
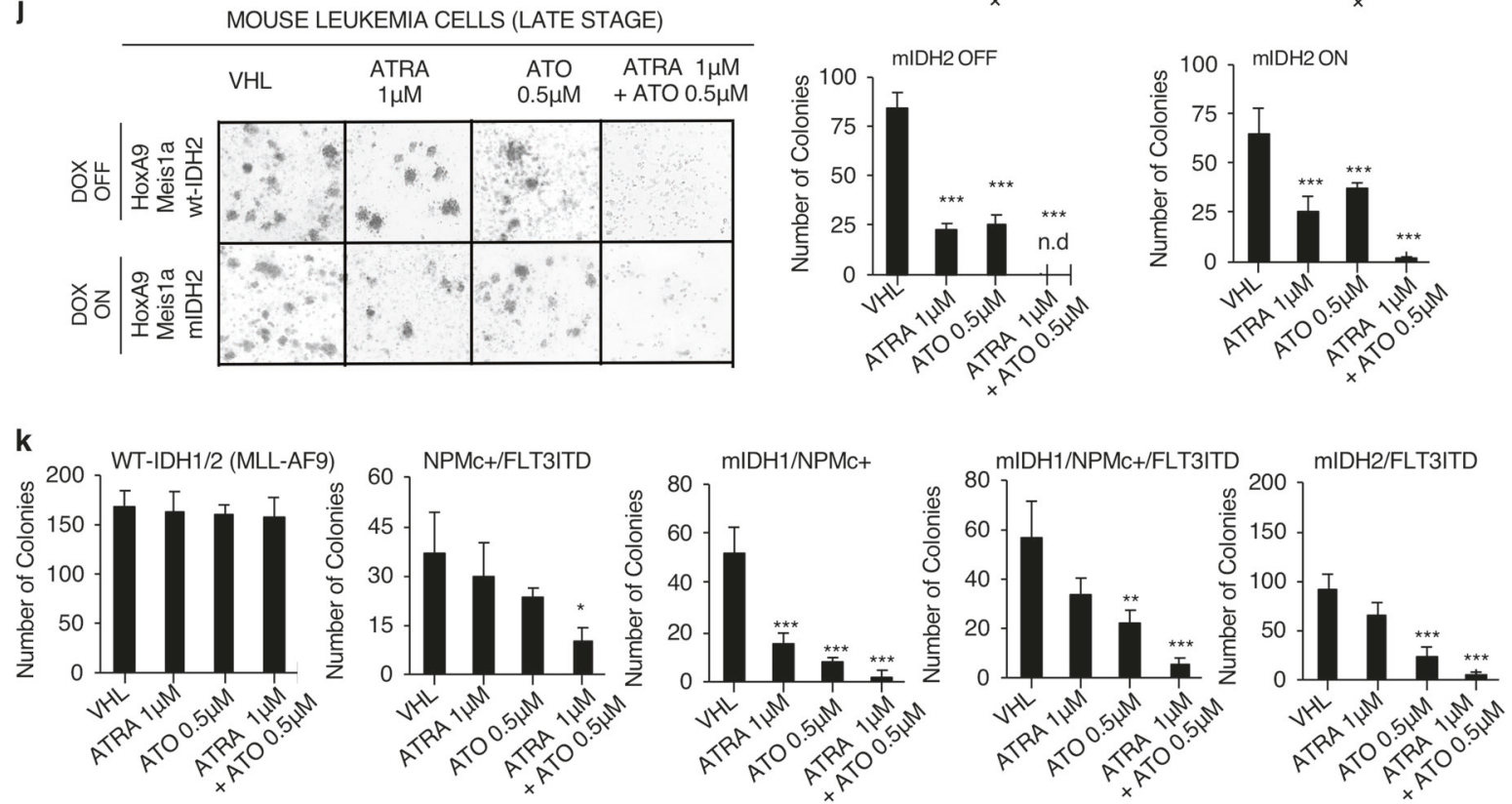

ATRA, demonstrating the power of this treatment combination to impact the severity of disease (Supplementary Information, Fig. S8h).

We next treated human primary AML cells isolated from a panel of patients (Supplementary Information, Fig. S9a and
Supplementary Information, Table S4): eleven harbouring an IDH2 mutation, three harbouring an IDH1 mutation, and seven with wild type IDH, with ATO/ATRA to evaluate their potential for clinical efficacy, as measured by colony formation in methylcellulose. Importantly, AML cells harbouring $\mathrm{mIDH} 2$ or $\mathrm{mIDH} 1$ formed 
Fig. $4 \mathrm{mIDH} 2$ leukaemia are sensitive to ATRA and ATO. a Schematic representation of the metastable state of HoxA9/Meis1a/mIDH2 cells: LSD1 activation sensitizes to ATRA, while the effect of this pro-differentiating stimuli is counteracted by high PIN1 levels. Treatment of mIDH2 cells with pharmacological doses of ATRA targets PIN1 for degradation and enables full LSD1 activation to promote differentiation. b-c, Cytospin images and quantitation of human TF1 cell line stably overexpressing the mutant variant R140Q of IDH2 (mIDH2) and the respective controls (CTRL) that have been treated with pharmacological concentrations of ATRA $\left(10^{-6} \mathrm{M}\right)$ or vehicle (DMSO). Data are means \pm SD; $t$-test; ${ }^{*} p \leq 0.05$. d Colony forming assay of mouse leukaemia cells harbouring mutant or wildtype IDH2 and treated with the PIN1 inhibitor (Juglone, $1 \mu \mathrm{M})$ or respective vehicle $(\mathrm{VHL})$. Colonies were quantitated 12 days after plating. Data are means \pm SD; one-way anova; ${ }^{* * *} p \leq 0.001$. e Colony forming assay of TF1 cells overexpressing the mIDH2 or respective control (CTRL) and treated with the PIN1 inhibitor (Juglone, $1 \mu \mathrm{M})$ or respective vehicle (VHL). Colonies were quantitated after 7 days from plating. Data are means \pm SD; one-way anova; ${ }^{* * *} p \leq 0.001$. $\mathbf{f}$ Schematic representation of the metastable state of HoxA9/Meis1a/mIDH2 cells. Increased one-carbon (1C-) metabolism and oxidative stress (ROS) sensitize cells to pro-oxidant agents such as arsenic trioxide (ATO). Excessive and impaired scavenging of ROS induces DNA damage and leads to cell death. The induction of 1C-metabolism and glutathione synthesis is essential to ensure survival of leukaemia cells. Thus, block of this antioxidant effect by treatment with ATO pushes cells towards collapse and death. g Fold induction of cell death (Annexin V/7AAD positive cells) in mouse leukaemia cells isolated from second or third recipients and treated in vitro with ATO $(0.5 \mu \mathrm{M})$ for $96 \mathrm{~h}$. Data are means \pm SD; $t$ test; ${ }^{*} p \leq 0.05$. h Fold induction of cell death (Annexin V/7AAD positive cells) in TF1 leukaemia cells overexpressing IDH $2^{\text {R140Q }}$ and treated with ATO $(0.5 \mu \mathrm{M})$ for $96 \mathrm{~h}$. Data are means \pm SD; $t$-test; ns not significant, ${ }^{*} p \leq 0.05$. i Representative images of methylcellulose colony forming assay and colony quantification of mouse leukaemia cells isolated from second transplants (early stage) and treated in vitro with pharmacological concentrations of ATRA $(1 \mu \mathrm{M})$, ATO $(0.5 \mu \mathrm{M})$, a combination of both (ATRA + ATO), or vehicle (VHL) as a control. Treatments were performed in either the presence or absence of the IDH mutation (CTRL). Data are mean \pm SD, one-way anova; ${ }^{* *} p \leq 0.01,{ }^{* * *} p \leq 0.001$. j Representative images of colony forming assay of HoxA9/Meis1A/mIDH2 mouse leukaemia cells isolated from third recipients (late stage) and treated with ATRA $(1 \mu \mathrm{M})$, ATO $(0.5 \mu \mathrm{M})$, a combination of both (ATRA + ATO), or vehicle (VHL) as a control. Histograms show the quantification of colony forming ability. Leukaemia cells were maintained on doxycycline (DOX ON) to favour the expression of the mIDH2 and increased $2 \mathrm{HG}$ levels or

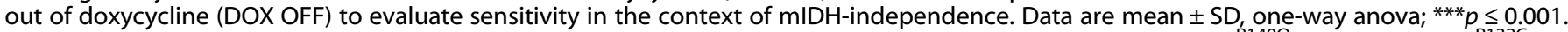
k Quantification of colony forming ability of mouse leukaemia cells harbouring mutations in IDH2 (IDH2 $\left.{ }^{\mathrm{R} 1400}\right)$ or IDH1 (IDH $1^{\mathrm{R} 132 \mathrm{C}}$ ) in association with multiple combinatorial genetic driver such as NPMc+ or FLT3ITD and treated with pharmacological concentrations of ATRA $(1 \mu \mathrm{M})$, ATO $(0.5 \mu \mathrm{M})$, a combination of both (ATRA + ATO), or vehicle (VHL) as a control. Non-mutant IDH2 cells such as MLL-AF9 or NPMc $+/$ /FLT3ITD were also included. Data are mean \pm SD; one-way anova; ${ }^{*} p \leq 0.05,{ }^{* *} p \leq 0.01,{ }^{* *} p \leq 0.001$. See also Supplementary Information, Figs. S4-S7

significantly reduced number of colonies upon treatment (Fig. 5a), and ANOVA analysis indicated a significant synergy in combination treatments (Fig. 5a and Supplementary Information Fig. S9a). Interestingly, in the case of patient \#AML_2, colonies arising from cells under single agent ATRA or ATO treatment gave primarily blast-like morphology, while the combination treatment resulted in almost complete loss of this morphology (Supplementary Information, Fig. S9b). Moreover, we tested one mIDH2 AML displaying de novo resistance to $\mathrm{mIDH} 2$ inhibitor (AG-221). Once again, this sample displayed PIN1 elevation and remarkable sensitivity to these agents (Supplementary Information, Fig. S10a-f).

Given the promising efficacy associated with this treatment in vitro, we next determined the impact of this treatment in vivo, using GEMM and human AML PDX models. Using third transplanted $\mathrm{mIDH} 2$ independent murine leukaemia cells (Supplementary Information, Fig. S10g), we established a pre-clinical trial to evaluate the efficacy of the ATRA/ATO combination in vivo. For each of the single treatments we observed a significant enhancement in survival of these mice, associated with reduction in bone marrow associated blast-like phenotype (Supplementary Information, Fig. S10h-j). Importantly however, whereas the combination treatment also led to an increased survival capacity over vehicle-treated mice (Supplementary Information, Fig. S10h), several of the mice treated with ATRA/ATO died not as a result of frank AML, but rather succumbed to differentiation syndrome (DS) characterized by infiltration of lungs with mature myeloid cells demonstrating strong myeloperoxidase (MPO) expression (Supplementary Information, Fig. S10k), a feature also associated with APL patients treated with ATRA. To further evaluate the efficacy of this treatment combination in AML harbouring $\mathrm{mIDH} 2$, we carried out two human PDX trials with two independent human primary AML cells (Fig. 5b, d). NSG mice transplanted with human primary mIDH2-positive AML cells were subjected to the treatment protocol, and to minimize onset of differentiation syndrome (DS), we utilized a metronomic dosing schedule, a protocol for treatment of APL patients. Importantly, for one PDX trial we observed a notable survival advantage for both ATO- and ATRAtreated mice, while combination-treated mice demonstrated a significant extension in the survival (Fig. 5b). Combination of ATO and ATRA as well as single agent treatments induced reduction of undifferentiated blasts and a significant increase in differentiating blasts in the bone marrow as evaluated by morphology (Fig. 5c and Supplementary Information, Fig. S11a). Our second PDX trial also demonstrated efficacy of ATO and the combination of ATRA and ATO with extensive differentiation of human blasts observed in the bone marrow at the time of euthanasia (Fig. 5e and Supplementary Information S11b). Importantly, for both PDX trials, we were able to detect a significant extension in the survival rate of the treated mice, which was not recapitulated by treatment of PDX model generated with AML cells harbouring wt-IDH1/2 genes (Supplementary Information, Fig. S11c). Moreover, for both of the PDX models generated with mutant IDH AML cells, and in particular the second case, aggressive DS was observed (Fig. 5f, g), highlighting the sensitivity of $\mathrm{mIDH}$ AML to combination of ATRA and ATO and a potential need for caution when treating human patients.

Thus, our data indicate that mIDH1/2-driven leukaemias are highly responsive to the classic APL treatment protocol, and differentiation therapy may represent a unique and complementary therapeutic approach for combination with $\mathrm{mIDH}$ targeting agents.

\section{DISCUSSION}

There is an acute need to develop rational combinations to treat disease and overcome resistance that arises in response to therapy. To facilitate this in the context of mutant IDH AML, we have sought to uncover the key mechanistic nodes that support maintenance of the disease and in doing so our efforts have identified mechanistic vulnerabilities associated with the expression of $\mathrm{mIDH} 2$ and subsequent evolution of this AML (Fig. 6). Critically, we extended our analysis and generalized our conclusions taking advantage of a number of $\mathrm{mIDH} 1 / 2$ mouse models of various genotypes, human $\mathrm{mIDH} 1 / 2$ cell lines, as well as primary mIDH1/2 AMLs.

Common outputs identified in the progression of $\mathrm{mIDH} 2 \mathrm{AML}$ include altered redox balance with ROS accumulation, genotoxic 
a

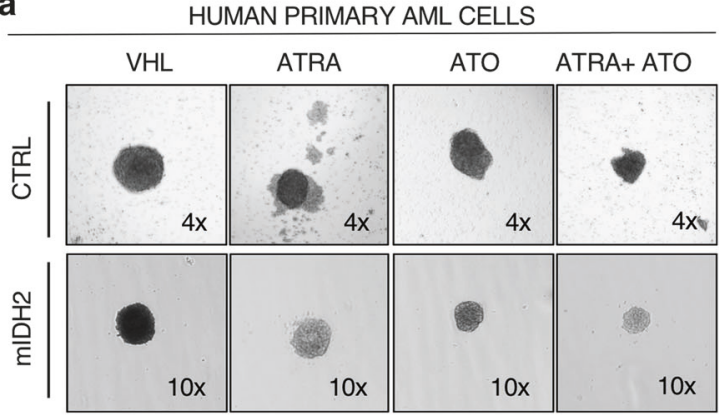

b

b PDX: IDH2R140Q; JAK2V617F; CRLF2V244M

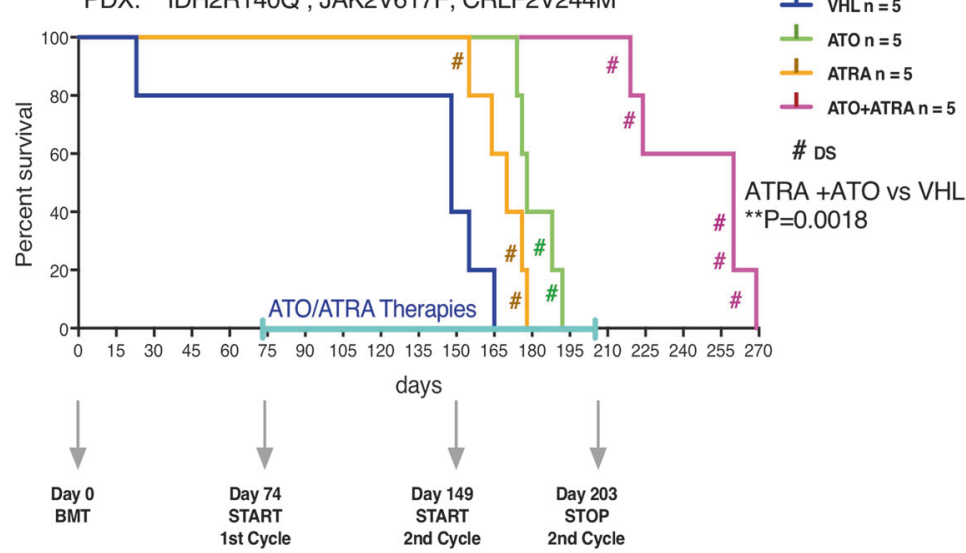

d

d PDX: IDH2R140Q

岁

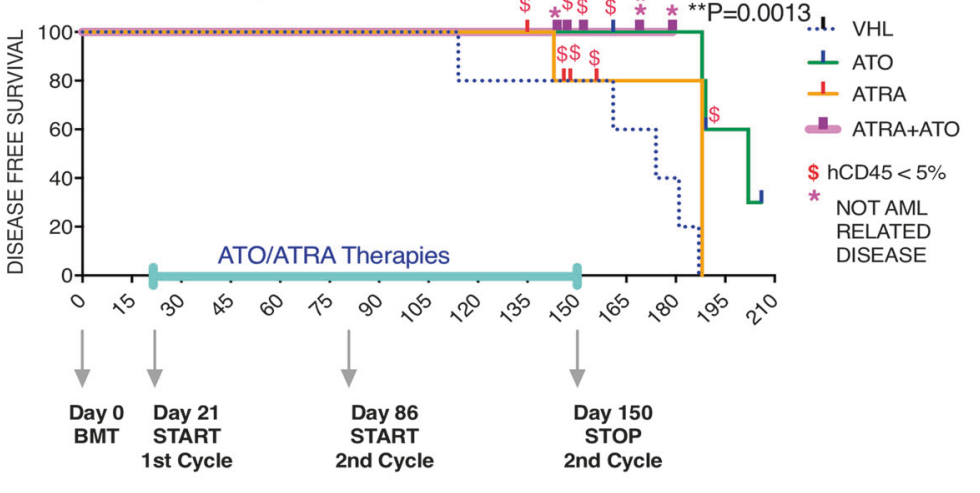

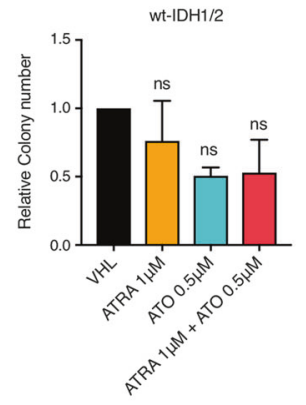

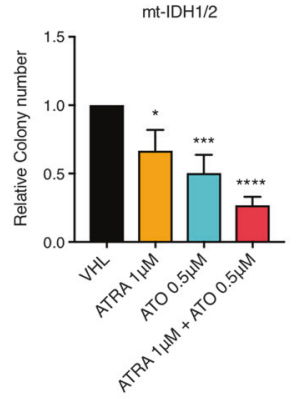

C

PDX IDH2 $2^{\mathrm{R} 140 \mathrm{Q}}$ AML (BM)

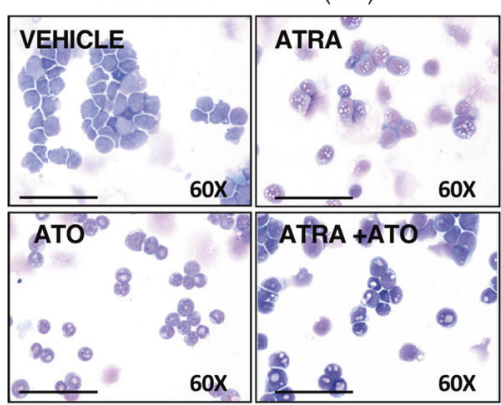

f

$$
\text { IHC MPO (LUNGS) }
$$

PDX IDH2 ${ }^{\mathrm{R} 140 Q} \mathrm{AML}-$ Fig.5b

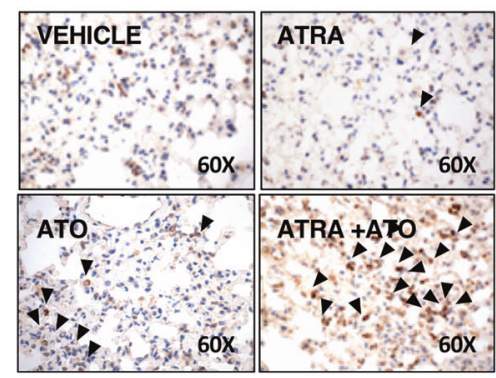

g IHC MPO (LUNGS)

PDX IDH2 ${ }^{\mathrm{R} 140 \mathrm{Q}} \mathrm{AML}-$ Fig.5d

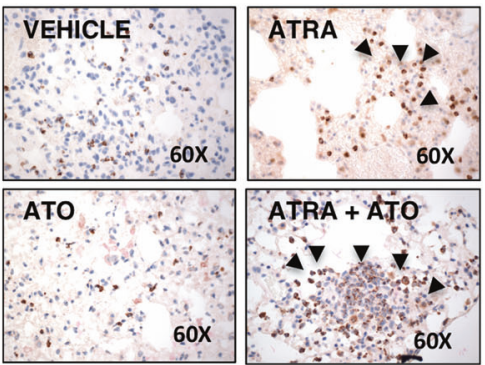

stress, genetic mutation and translocation, MAPK/PI3K hyperactivation, and an aberrant ATRA transcriptional program (Fig. 6). Indeed, the relevance of MAPK dependence and relevance of differentiation in therapy has recently been highlighted for patients treated with Enasidenib, thereby validating the molecular underpinnings of our model. ${ }^{8}$
Importantly, we show that mIDH2-dependent molecular and metabolic rewiring can drive ROS accumulation, and a dependence on PIN1 with the prolyl isomerase acting as a critical node in mediating a differentiation block in spite of the increased ATRA sensitization mediated by the inactivation of LSD1. While PIN1 is proto-oncogenic in its action triggering the activation of MAPK/ 
Fig. 5 Combined ATRA and ATO treatment eradicates mIDH2 leukaemia. a Methylcellulose colony forming assay and colony quantification of human primary $\mathrm{mIDH} 2 \mathrm{AML}$ blasts treated in vitro with pharmacological concentrations of ATRA $(1 \mu \mathrm{M})$, ATO $(0.5 \mu \mathrm{M})$, a combination of both (ATRA + ATO), or vehicles (VHL) as control. Treatments were performed in either the presence of mIDH2 or absence of the mutation (CTRL). Data are mean $\pm \mathrm{SD}$; one-way anova; ns not significant, ${ }^{*} p \leq 0.05,{ }^{* * *} p \leq 0.001,{ }^{* * *} p \leq 0.0001$. b, d Kaplan-Meyer curve showing the percentage of disease free survival related to ATO/ATRA pre-clinical trial on mIDH2 PDX. $n=2$ different mIDH2 AMLs were isolated from bone marrow at diagnosis. Data were analysed by Log-Rank (Mentel-Cox) test. Statistics associated with mIDH2 AML PDX shown in b ATRA + ATO vs VHL $p=0.0018$. Statistics associated with mIDH2 AML PDX shown in c ATRA + ATO vs VHL $p=0.0013$. \#DS: mice show differentiation syndrome (DS) (aka. ATRA syndrome). c, e Representative cytospin images showing cells isolated from BM of PDX mice treated with ATRA and ATO. $\mathbf{f}, \mathbf{g}$, Representative images of IHC for the differentiation marker human myeloperoxidase (MPO) on lung tissues isolated from PDX mice treated with ATRA and ATO or VEHICLE solutions. Accumulation of human MPO-positive cells is a major characteristic of DS. mIDH2: IDH2 ${ }^{\text {R140Q; }}$ BM bone marrow, ATRA retinoic acid, ATO arsenic Trioxide. See also Supplementary Information, Figs. S8-S12 and Supplementary Information, Table S4

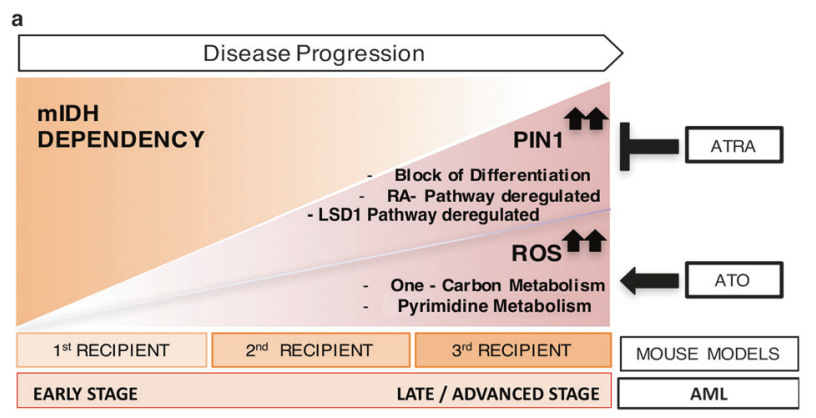

Fig. 6 Scheme of ATO and ATRA targeting in $\mathrm{mIDH}$ leukaemia. Scheme of leukaemia evolution from $\mathrm{mIDH}$ dependent to $\mathrm{mIDH} 2$ independent states (e.g. acquisition of resistance to $\mathrm{mIDH}$ inhibition). Multiple alterations including metabolic reprogramming, and transcriptional rewiring are acquired during progression and coexist in the $\mathrm{mIDH} 2$ independent stage. In this multifactorial setting, we unveil PIN1, deregulated LSD1 signaling and high ROS levels as key factors for upregulated pro-survival and proliferation programs (MAPK/PI3K pathways), alterations to functional ATRA signalling, and altered metabolic state (one-carbon metabolism and increased nucleic acid synthesis). These features represent targetable vulnerabilities of leukaemia cells and highlight the potential for using ATO (pro-oxidant) and ATRA (pro-differenting) drugs. Most importantly, sensitivity to ATO and ATRA is a vulnerability already present at an early stage in $\mathrm{mIDH} 2$-dependent leukaemia, which is mantained in later stages during the disease progression

$\mathrm{PI} 3 \mathrm{~K}$ signaling, it is also known to trigger ROS production through mitochondrial activation. ${ }^{39-41}$ As leukemic blasts are known to be particularly sensitive to ROS levels, this in turn creates an inherent vulnerability, which negatively impacts stem cell maintenance and facilitates exhaustion. ${ }^{42}$ Indeed, our data illustrate how natural selection towards an $\mathrm{mIDH} 2$-independent state further exacerbates a PIN1-ATRA block and the altered cellular balance towards extreme levels of oxidation.

On this basis, we identify the classical targeted combination therapy of APL, ATRA and ATO, as a therapeutic strategy for the treatment of $A M L$ harboring mutation in $\mathrm{mIDH} 1 / 2$, independent of multiple and diverse additional genetic events. This is highlighted on the one hand by the exquisite sensitivity to this combination therapy of our multiple leukaemic models harbouring $\mathrm{mIDH}$ along with other established driver mutations (e.g. NPMc + and FLT3-ITD), and on the other hand by the fact that ATRA/ATO is an effective combination independent of the evolutionary stage of the mIDH AML. Such an approach is of utmost relevance in view of a recent study from Quek et al. on clonal heterogeneity and evolution of mIDH2 AML treated with Enasidenib, demonstrating that multiple resistance mechanisms driven by a diverse mutational landscape might be at play to restore the block in differentiation, highlighting the need for additional therapeutic approaches to overcome such resistance. $^{12}$
In APL, the ATRA/ATO combination selectively targets the PIN1PML-RARa oncogenic node, ${ }^{43}$ while in mIDH-leukaemia, the ATRA/ ATO combination treatment selectively targets the oncogenic mIDH-PIN1-LSD1 node. In addition, using a cohort of primary human AML cells that harbor either $\mathrm{mIDH} 1$ or $\mathrm{mIDH} 2$, we have been able to clearly demonstrate the potential efficacy of the ATRA/ATO combination to treat this subset of AML. Our analysis suggest that these oncogenes sensitize to the effects of ATRA plus ATO. Thus, the combination of these agents with specific mIDH targeting agents may in turn potentiate and extend the efficacy of these inhibitors. Indeed, the fact that mIDH blocks myeloid differentiation and the ATRA response similarly to PML-RARa, also suggests that rare atypical APL cases lacking the usual PML/RARa translocations may actually harbor mutation in IDH enzymes. Intriguingly, in keeping with this notion, out of two atypical APL we analyzed, one displayed mutations in IDH2 enzyme (Supplementary Information, Fig. S12). Furthermore, mutations in IDH1/2 have been described for AMLs displaying an acute promyelocytic leukaemia-like (APL-like) immunophenotype by flow cytometry, ${ }^{44}$ reinforcing the idea that $A P L$ and $A M L s$ carrying $\mathrm{mIDH}_{2}$ harbor common pathways of evolution and vulnerabilities despite distinct genetic drivers.

Taken together, this study provides critical understanding of the mechanisms underlying $\mathrm{mIDH}$-driven $\mathrm{AML}$ pathogenesis, and underscores the value and insights that can be gained from modeling cancer dependencies and vulnerabilities in appropriate model systems. Critically, our data point towards the development of a rationale-based therapeutic strategy for the targeted and effective treatment of leukaemia.

\section{MATERIALS AND METHODS}

Cells

The human leukaemia U937 and TF1 cell lines were purchased from the American Type Culture Collection (ATCC; https://www. atcc.org). The U937 cells were maintained in RPMI supplemented with $10 \%$ FBS and $1 \%$ penicillin/streptomycin. The TF1 cells were maintained in RPMI-1640 supplemented with L-glutamine (Gibco), $10 \%$ FBS, $1 \%$ penicillin / streptomycin, human GM-CSF $(2 \mathrm{ng} / \mathrm{mL})$ $(R \& D)$. Both cell culture protocols were in accordance with American Type Culture Collection guidelines (ATCC; https://www. atcc.org).

Human primary AML cells isolated from patients at diagnosis were provided by BioBanco LAFe (Spain) (http://www.iislafe.es/ biobanco-la-fe.aspx), Dana Farber Cancer Institute (DFCI) Leukaemia/Lymphoma Xenograft Core (LLX) (https://www.proxe.org) or by co-authors David Avigan, Dina Stroopinsky, Lourdes Mendez (Beth Israel Deaconess Medical Center - BIDMC, Boston, MA USA). Primary AML cells were isolated from patients' bone marrow tissues or peripheral blood and grown in H4434 methylcellulose medium (MethoCult ${ }^{\mathrm{TM}} \mathrm{H} 4434$ Classic, Stem Cell Technologies, Catalog \#04444) or QBSF-60 medium (VWR) supplemented with human FLT3 ligand $(10 \mathrm{ng} / \mathrm{mL})$, TPO $(20 \mathrm{ng} / \mathrm{mL})$, IL-3 $(10 \mathrm{ng} / \mathrm{mL})$, 
458

MEM $1 \times$ (Sigma-Aldrich), 2\% penicillin and streptomycin, 20\% High grade bovine serum. All primary AML cells were then processed for methylcellulose assay with reagent treatments as described.

\section{Vectors and constructs}

To generate stable lines for mutant IDH expression, TF1 and U937 cells were infected with lenti-virus pLVX-IRES - neo vector (Clontech \#6321810) or pCIG3 (pCMV-IRES_GFP, from Felicia Goodrum, Addgene plasmid \# 78264), or retro-virus pBABE-GFP (from William Hahn, Addgene plasmid \# 10668) containing full length IDH2/R140Q, IDH1/R132C or respective empty vectors as controls. Full length human PIN1 cDNA (MGC, Dharmacon) was amplified and cloned into the EcoRI site of the lentiviral vector pCIG3 (from Felicia Goodrum, Addgene plasmid \# 78264). Lentiviral particles for stable silencing of PIN1 and relative control were from Sigma (MISSION Lentiviral Transduction Particles, SHC016V-1EA, SHCLNV: NM_006221, CloneID: TRCN0000010577).

Murine studies and animal housing

The tetracycline-inducible IDH2 ${ }^{\mathrm{R} 140 \mathrm{Q}}$ mouse model was previously created in our lab and backcrossed into the C57BL/6 J background as previously described. ${ }^{13}$ To activate transgene expression in vivo, a doxycycline-containing chow (Harlan Laboratories) was administered to animals at the time of weaning ( 3 weeks of age). To stop transgene expression, mice were taken off the doxycycline-containing diet and placed on a regular laboratory rodent diet. We obtained approval for all animal experiments from the Beth Israel Deaconess Medical Center (BIDMC) and the Institutional Animal Care and Use Committee (IACUC) (Research Protocol \#076-2017).

\section{Isolation of RNA and DNA and Next-generation sequencing}

For RNA-Seq, total RNA was isolated from a pure population of sorted leukaemia cells according to previously described methods. ${ }^{45}$ DNAse treatment was performed to eliminate traces of genomic DNA. For WES, genomic DNA was prepared using the QUIAGEN DNeasy Blood \& Tissue kit. Nucleic acids (DNA and RNA) were quantitated by Nanodrop (Thermo Scientific). NGS services were performed by the Molecular Biology Core Facilities at Dana Farber Cancer Institute.

\section{Reactive oxygen species analysis}

Live cells $\left(25 \times 10^{4}\right.$ cells) were incubated with $2.5 \mu \mathrm{M}$ MitoSOX ${ }^{\mathrm{TM}}$ Red mitochondrial superoxide indicator for live-cell imaging (Invitrogen, Catalog \#M36008) or CellROX ${ }^{\circledR}$ Deep Red Reagent or CellROX Deep Red Reagent (Invitrogen, Catalog \#C10422), processed according to manufacture protocols and analysed as previously described. ${ }^{46}$

\section{Immunoblotting}

For western blotting, leukaemia cells were lysed in RIPA buffer (Sigma) supplemented with protease and phosphatase inhibitors. Proteins were separated on 4-12\% Bis-Tris gradient gels (Invitrogen), then transferred to polyvinylidine difluoride membranes (Immobilon P, Millipore) as previously described, ${ }^{47}$ and probed with the indicated antibodies diluted (1:1000 or 1:500) in $1 \times$ PBS with $5 \%$ BSA and $0.1 \%$ Tween-20. Antibodies used in this study: Mouse monoclonal Anti-IDH2(R140Q) (NewEast Biosciences, \#26165), Rabbit anti-RARalpha (Cell Signaling Technology, 2554 P; Santa Cruz Biotechnology, sc-551), Rabbit anti-RXRalpha (D6H10) (Cell Signaling Technology, 3085 P), Rabbit anti-CEPBE (abcam, ab64970), Rabbit anti-C/EBPalpha (p42) (Cell Signaling Technology, 2843 S), Mouse anti-HSP90 (BD Biosciences, BDB610419), Rabbit anti - GAPDH (14C10) (Cell Signaling Technology, \#2118).
Quantification and statistical analysis

Statistical analysis was performed using GraphPad Prism7 software. One-way anova or $t$-test were used as appropriate. The Logrank (Mentel-Cox) statistical analysis was used to evaluate significance on survival curves.

Additional detailed materials and methods can be found in the Supplementary Information, Data S1.

\section{ACKNOWLEDGEMENTS}

We thank all members of the Pandolfi laboratory for critical comments. Lauren Southwood and Elizabeth Stack for editing the manuscript. We are grateful to Markus Reschke, Akinobu Matsumoto, Nicole Pandell, Valerie Maymi, John Asara, John F. Daley and Zach Herbert for technical support with experiments. We are also thankful to Jacqueline Fung and Jesse M. Katon for their technical help and to Kun Ping Lu for support and reagents. V.M. was supported by EMBO Long Term post-doctoral fellowship (ALTF 1011-2014), European Commission (LTFCOFUND2013, GA2013-609409) and by the Leukaemia and Lymphoma Society (Career Development Program, Grant number: 5437-16). R.P. was partially supported by Marie Curie Action Fellowship "Mobility Program for post graduated scientists in Life Sciences" (C. I.13001124001202512454). E.M. is supported by the Società Italiana di Biofisica e Biologia Molecolare (SIBBM). This work was supported in part by the Ludwig Center at Harvard and $\mathrm{NIH} / \mathrm{NCl}$ grants R35CA197529. and R01CA142874 to P.P.P.

\section{AUTHOR CONTRIBUTIONS}

P.P.P., V.M. and J.G.C. conceived the hypothesis, planned and designed the experiments and wrote the paper. L.L. managed the study. V.M. performed in vivo and in vitro experiments regarding mouse, human leukaemia primary cells and cell lines. V.M. engineered TF1 cell line for Pin1 overexpression or silencing. V.M and R.P. engineered the U937 and TF1 cell lines for the stable expression of the mutant variants $\mathrm{R} 140 \mathrm{Q}$ of $\mathrm{IDH} 2$ and $\mathrm{R} 132 \mathrm{C}$ of $\mathrm{IDH} 1$ and performed in vivo and in vitro experiments, real-time qRT-PCR and FACS analysis. M.C. performed western blot analyses on mouse and human leukaemia cells. G.C. performed colony assays on human mIDH AML. V.M and J.G.C. performed the pre-clinical trials, O.P. performed histopathological analyses, A.V.M., J.G.C., L.M., D.S., J.G., D.A., J.C.Z. and F.L. contributed to in vitro and in vivo experiments. J.C.A., A.L., R.M.S., I.G., D.S., D.A. and J.C.Z. provided human primary patient AML samples. E.M., J.D.L. and M.K.B. performed the bioinformatics analysis of the data.

\section{ADDITIONAL INFORMATION}

Supplementary information accompanies this paper at https://doi.org/10.1038/ s41422-019-0162-7.

Competing interests: P.P.P. is a member of the Scientific Advisory Board of Agios Pharmaceuticals, Cambridge, MA. P.P.P., V.M., J.G.C. and M.C. have filed a U.S. Patent Application No. 62/593,761, entitled "ARSENIC TRIOXIDE AND RETINOIC ACID COMPOUNDS FOR TREATMENT OF IDH2-ASSOCIATED DISORDERS", filed December 1,2017 . This application is still pending. The remaining authors declare no competing interests.

\section{REFERENCES}

1. Chen, $C$. et al. Cancer-associated IDH2 mutants drive an acute myeloid leukemia that is susceptible to Brd4 inhibition. Genes Dev. 27, 1974-1985 (2013).

2. Fujii, T., Khawaja, M. R., DiNardo, C. D., Atkins, J. T. \& Janku, F. Targeting isocitrate dehydrogenase (IDH) in cancer. Discov. Med. 21, 373-380 (2016).

3. Losman, J. A. \& Kaelin, W. G. Jr. What a difference a hydroxyl makes: mutant IDH, (R)-2-hydroxyglutarate, and cancer. Genes Dev. 27, 836-852 (2013).

4. Garraway, L. A. \& Lander, E. S. Lessons from the cancer genome. Cell 153, 17-37 (2013).

5. Chan, S. M. et al. Isocitrate dehydrogenase 1 and 2 mutations induce BCL-2 dependence in acute myeloid leukemia. Nat. Med. 21, 178-184 (2015).

6. Clark, O., Yen, K. \& Mellinghoff, I. K. Molecular pathways: isocitrate dehydrogenase mutations in cancer. Clin. Cancer Res. 22, 1837-1842 (2016).

7. Dang, L., Yen, K. \& Attar, E. C. IDH mutations in cancer and progress toward development of targeted therapeutics. Ann. Oncol. 27, 599-608 (2016).

8. Amatangelo, M. D. et al. Enasidenib induces acute myeloid leukemia cell differentiation to promote clinical response. Blood 130, 732-741 (2017).

9. Holohan, C., Van Schaeybroeck, S., Longley, D. B. \& Johnston, P. G. Cancer drug resistance: an evolving paradigm. Nat. Rev. Cancer 13, 714-726 (2013). 
10. Stein, E. M. et al. Enasidenib in mutant IDH2 relapsed or refractory acute myeloid leukemia. Blood 130, 722-731 (2017).

11. Intlekofer, A. M. et al. Acquired resistance to IDH inhibition through trans or cis dimer-interface mutations. Nature 559, 125-129 (2018).

12. Quek, L. et al. Clonal heterogeneity of acute myeloid leukemia treated with the IDH2 inhibitor enasidenib. Nat. Med. 24, 1167-1177 (2018).

13. Kats, L. M. et al. Proto-oncogenic role of mutant IDH2 in leukemia initiation and maintenance. Cell Stem Cell 14, 329-341 (2014).

14. Mentch, S. J. \& Locasale, J. W. One-carbon metabolism and epigenetics: understanding the specificity. Ann. N. Y. Acad. Sci. 1363, 91-98 (2016).

15. Sykes, D. B. et al. Inhibition of dihydroorotate dehydrogenase overcomes differentiation blockade in acute myeloid leukemia. Cell 167, 171-186 e115 (2016).

16. Couto, N., Wood, J. \& Barber, J. The role of glutathione reductase and related enzymes on cellular redox homoeostasis network. Free Radic. Biol. Med. 95, 27-42 (2016).

17. Noguera, N. I. et al. High-dose ascorbate and arsenic trioxide selectively kill acute myeloid leukemia and acute promyelocytic leukemia blasts in vitro. Oncotarget $\mathbf{8}$ 32550-32565 (2017).

18. Granito, A. et al. PML nuclear body component Sp140 is a novel autoantigen in primary biliary cirrhosis. Am. J. Gastroenterol. 105, 125-131 (2010).

19. Zucchelli, C. et al. Structure of human Sp140 PHD finger: an atypical fold interacting with Pin1. Febs. J. 281, 216-231 (2014).

20. Gombart, A. F. \& Koeffler, H. P. Neutrophil specific granule deficiency and mutations in the gene encoding transcription factor C/EBP(epsilon). Curr. Opin. Hematol. 9, 36-42 (2002).

21. Friedman, A. D. C/EBPalpha in normal and malignant myelopoiesis. Int. J. Hematol. 101, 330-341 (2015).

22. Patial, S. et al. Enhanced stability of tristetraprolin mRNA protects mice against immune-mediated inflammatory pathologies. Proc. Natl Acad. Sci. USA 113, 1865-1870 (2016).

23. Cauchy, P. et al. Chronic FLT3-ITD signaling in acute myeloid leukemia is connected to a specific chromatin signature. Cell Rep. 12, 821-836 (2015).

24. Schenk, T. et al. Inhibition of the LSD1 (KDM1A) demethylase reactivates the alltrans-retinoic acid differentiation pathway in acute myeloid leukemia. Nat. Med 18, 605-611 (2012)

25. Martens, J. H. et al. PML-RARalpha/RXR alters the epigenetic landscape in acute promyelocytic leukemia. Cancer Cell 17, 173-185 (2010).

26. Boutzen, $\mathrm{H}$. et al. Isocitrate dehydrogenase 1 mutations prime the all-trans retinoic acid myeloid differentiation pathway in acute myeloid leukemia. J. Exp. Med. 213, 483-497 (2016)

27. Wang, F. et al. Targeted inhibition of mutant $\mathrm{IDH} 2$ in leukemia cells induces cellular differentiation. Science 340, 622-626 (2013).

28. Gianni, M. et al. Inhibition of the peptidyl-prolyl-isomerase Pin1 enhances the responses of acute myeloid leukemia cells to retinoic acid via stabilization of RARalpha and PML-RARalpha. Cancer Res. 69, 1016-1026 (2009).

29. Wei, S. et al. Active Pin1 is a key target of all-trans retinoic acid in acute promyelocytic leukemia and breast cancer. Nat. Med. 21, 457-466 (2015).

30. Brondani, V., Schefer, Q., Hamy, F. \& Klimkait, T. The peptidyl-prolyl isomerase Pin1 regulates phospho-Ser77 retinoic acid receptor alpha stability. Biochem. Biophys. Res. Commun. 328, 6-13 (2005).
31. Marchwicka A., Marcinkowska E. Regulation of expression of CEBP genes by variably expressed vitamin $D$ receptor and retinoic acid receptor alpha in human acute myeloid leukemia cell lines. Int. J. Mol. Sci. 19, 50-63 (2018).

32. Grignani, F. et al. The acute promyelocytic leukemia-specific PML-RAR alpha fusion protein inhibits differentiation and promotes survival of myeloid precursor cells. Cell 74, 423-431 (1993).

33. Testa, U. et al. PML/RAR alpha + U937 mutant and NB4 cell lines: retinoic acid restores the monocytic differentiation response to vitamin D3. Cancer Res. 54, 4508-4515 (1994).

34. Ghaffari, S. H. et al. Cytotoxic effect of arsenic trioxide on acute promyelocytic leukemia cells through suppression of NFkbeta-dependent induction of hTERT due to down-regulation of Pin1 transcription. Hematology 17, 198-206 (2012).

35. Kozono, S. et al. Arsenic targets Pin 1 and cooperates with retinoic acid to inhibit cancer-driving pathways and tumor-initiating cells. Nat. Commun. 9, 3069 (2018).

36. Reineke, E. L. et al. Degradation of the tumor suppressor PML by Pin 1 contributes to the cancer phenotype of breast cancer MDA-MB-231 cells. Mol. Cell. Biol. 28, 997-1006 (2008).

37. Yang D. et al. A novel controlled release formulation of the Pin 1 inhibitor ATRA to improve liver cancer therapy by simultaneously blocking multiple cancer pathways. J. Control Release 2018;269:405-422.

38. Forghieri, F. et al. All-trans retinoic acid (ATRA) in non-promyelocytic acute myeloid leukemia (AML): results of combination of ATRA with low-dose Ara-C in three elderly patients with NPM1-mutated AML unfit for intensive chemotherapy and review of the literature. Clin. Case Rep. 4, 1138-1146 (2016).

39. Costantino, S., Paneni, F., Luscher, T. F. \& Cosentino, F. Pin1 inhibitor Juglone prevents diabetic vascular dysfunction. Int. J. Cardiol. 203, 702-707 (2016).

40. Boussetta, T. et al. The prolyl isomerase Pin1 acts as a novel molecular switch for TNF-alpha-induced priming of the NADPH oxidase in human neutrophils. Blood 116, 5795-5802 (2010)

41. Pinton, $P$. et al. Protein kinase $C$ beta and prolyl isomerase 1 regulate mitochondrial effects of the life-span determinant p66Shc. Science 315, 659-663 (2007).

42. Carracedo, A., Cantley, L. C. \& Pandolfi, P. P. Cancer metabolism: fatty acid oxidation in the limelight. Nat. Rev. Cancer 13, 227-232 (2013).

43. de The, H., Pandolfi, P. P. \& Chen, Z. Acute promyelocytic leukemia: a paradigm for oncoprotein-targeted cure. Cancer Cell 32, 552-560 (2017).

44. Mason, E. F., Kuo, F. C., Hasserjian, R. P., Seegmiller, A. C. \& Pozdnyakova, O. A distinct immunophenotype identifies a subset of NPM1-mutated AML with TET2 or IDH1/2 mutations and improved outcome. Am. J. Hematol. 93, 504-510 (2018).

45. Chevillard, S. A method for sequential extraction of RNA and DNA from the same sample, specially designed for a limited supply of biological material. Biotechniques 15, 22-24 (1993).

46. Mugoni, V. et al. Ubiad1 is an antioxidant enzyme that regulates eNOS activity by CoQ10 synthesis. Cell 152, 504-518 (2013).

47. Reschke, M. et al. Characterization and analysis of the composition and dynamics of the mammalian riboproteome. Cell Rep. 4, 1276-1287 (2013). 